\title{
The Effects of Canopy Leaf Area Index on Airflow Across Forest Edges: Large-eddy Simulation and Analytical Results
}

\author{
M. Cassiani · G. G. Katul • J. D. Albertson
}

Received: 7 February 2007 / Accepted: 12 November 2007 / Published online: 6 December 2007

(C) Springer Science+Business Media B.V. 2007

\begin{abstract}
The structure of turbulent flows along a transition between tall-forested canopies and forest clearings continues to be an active research topic in canopy turbulence. The difficulties in describing the turbulent flow along these transitions stem from the fact that the vertical structure of the canopy and its leaf area distribution cannot be ignored or represented by an effective roughness length. Large-eddy simulation (LES) runs were performed to explore the effect of a homogeneous variation in the forest leaf area index (LAI) on the turbulent flow across forest edges. A nested grid numerical method was used to ensure the development of a deep boundary layer above the forest while maintaining a sufficiently high resolution in the region close to the ground. It was demonstrated that the LES here predicted first-order and second-order mean velocity statistics within the canopy that agree with reported Reynolds-Averaged Navier-Stokes (RANS) model results, field and laboratory experiments. In the simulations reported here, the LAI was varied between 2 and 8 spanning a broad range of observed LAI in terrestrial ecosystems. By increasing the forest LAI, the mean flow properties both within the forest and in the clearing near the forest edge were altered in two fundamental ways: near the forest edge and into the clearing, the flow statistical properties resembled the so-called back-facing step (BFS) flow with a mean recirculation zone near the edge. Another recirculation zone sets up downstream of the clearing as the flow enters the tall forest canopy. The genesis of this within-forest recirculation zone can be primarily described using the interplay between the mean pressure gradients (forcing the flow) and the drag force (opposing the flow). Using the LES results, a simplified analytical model was also proposed to explain the location of the recirculation zone inside the canopy and its dependence on the forest LAI. Furthermore, a simplified scaling argument that decomposes the mean velocity at the outflow edge into a superposition of 'exit flow' and BFS-like flow with their relative importance determined by LAI was explored.
\end{abstract}

M. Cassiani $(\varangle) \cdot$ G. G. Katul · J. D. Albertson

Department of Civil and Environmental Engineering, Duke University, 121 Hudson Hall, P. O. Box 90287,

Durham, NC 27708, USA

e-mail: massimo.cassiani@duke.edu

G. G. Katul · J. D. Albertson

Nicholas school of the Environment, Duke University, Durham, NC 27708, USA 
Keywords Back-facing step flow · Boundary layer · Exit flow · Forest edge . Large-eddy simulations $\cdot$ Recirculation

\section{Introduction}

Recent studies have shown that fragmented landscapes are becoming one of the most widespread features of the modern world (Laurence 2004) with important consequences to landatmosphere interactions. On a local scale, (i.e. $<1 \mathrm{~km}$ ), such fragmentation influences the up-scaling of fluxes and meteorological states from patchy surfaces (Klaassen 1992; Albertson and Parlange 1999a, b) and affects a large number of biotic and abiotic processes, including tree mortality, seed and pollen deposition in gaps (with direct consequences to plant community development and gene flow) and enhanced dry deposition of atmospheric pollutants. An abrupt roughness transition from one extensive uniform region to another, where the discontinuity is a well-defined edge normal to the wind direction, represents the simplest and yet most fundamental of surface changes in fragmented landscapes and serves as a starting point for exploring how the bulk flow properties adjust to such disturbances.

Given its importance, it is of no surprise that early laboratory (Antonia and Luxton 1974) and field (e.g. Raynor 1971; Bergen 1975) experiments, as well as second-order closure modelling studies (e.g. Rao et al. 1974), focused on the growth of the internal boundary layer, a region defined by the influence of the abrupt jump in the surface shear stress due to a jump in the aerodynamic roughness length (see the review in Garratt 1990). Albertson and Parlange (1999a, b) used a large-eddy simulation (LES) to investigate the effects of small changes of roughness on the vertical momentum flux. However, transitions between tall-forested canopies and forest clearings present an added level of complexity because the 'morphological' signature of the forest on the flow disturbance at downstream distances cannot be ignored and a single roughness length cannot represent its effect.

Over the past three decades, a number of field experiments have reported observations on mean flow properties near tall-forested edges, though their spatial sampling remains rather restricted to a few tower locations and a few profile levels (Irvine et al. 1997; Flesch and Wilson 1999; Detto et al. 2007). LES has now sufficiently matured to permit exploring the effects of forest edges on flow disturbances within the canopy at sufficient spatial resolution. Recent evidence of such a success is the agreement between LES computations reported in Yang et al. (2006a, b) and the TKE component budget measurements in a wind tunnel reported by Morse et al. (2002). Moreover, LES have been used to explore a similar problem, flow behind shelterbelts (Patton et al. 1998), with satisfactory agreement with measurements reported in Judd et al. (1996).

In reality even within the restricted scope of an idealized abrupt transition across an extensive forest-clearing interface, a large number of processes such as topography, atmospheric stability, patch sizes, upper atmospheric conditions and boundary-layer depth, threedimensional distribution of the foliage and tree spacing all confound general conclusions. However, exploring the joint effect of all these processes is well beyond the scope of a single study.

The effect of increasing forest LAI on the bulk flow properties within the forest canopy and the clearing is among the least explored variables despite direct and indirect evidence of the large impact of LAI on the basic structure of the mean flow field. For example, early smoke release experiments by Bergen (1975) reported recirculation zones near a forest-grass interface, results confirmed by wind-tunnel studies in Raupach et al. (1987) who also reported the onset of 'rotor-like' motion near the edge. Field experiments by Irvine et al. (1997) did 
not reveal any recirculation zone, while Flesch and Wilson (1999) found an intermittent recirculation but concluded that the flow could be described as exit flow rather than a recirculating one. However, using Reynolds-Averaged Navier-Stokes (RANS) model calculations, the same authors (Wilson and Flesch 1999) found a substantial recirculation zone close to the ground. Our comparison with the work of these two authors showed that a mean adverse flow possibly existed close to the ground. Liu et al. (1996) concluded that an intermittent recirculation was present in the wind-tunnel experiment of Chen et al. (1995), and using a $k-\varepsilon$ RANS model they showed that this recirculation was dependent on the forest LAI. Using detailed field experiments on the mean momentum budget near a dense and sharp forest edge, Detto et al. (2007) argued that an intermittent recirculation zone may exist given the similarities between the flow from a dense forest into a clearing and the back-facing step (BFS) flow. However, only indirect evidence in support of this analogy was provided because their gradient measurements were conducted at only a single point near the forest edge. It is clear that the interplay between the forest LAI and the onset of a recirculation zone remains an open problem.

Since analogies to BFS flows are used here, the main characteristics of this kind of turbulent flow are briefly described. Turbulence in BFS flows is generally produced by a mean velocity gradient perpendicular to the main flow direction. Depending on what causes this velocity gradient, two types of turbulence regimes, both present in BFS flows, are often distinguished: (i) free turbulence, and (ii) wall turbulence. The free turbulence occurs immediately downstream of the step and is produced by the fact that a mass of fluid with relative high velocity is forced to move along a mass of fluid with a relative low velocity (such as the recirculation zone). The wall turbulence occurs in the vicinity of the bottom wall. Often labelled as the simplest reattaching flow (e.g. Simpson 1989), the mean and turbulent flow fields of BFS remain fairly complex because the mean momentum balance is governed by a number of processes, including mean pressure gradients, advective terms, and turbulent stresses. In BFS, the upstream boundary layer detaches at the sharp corner and a mixing layer with intense shear stresses (free-shear layer) develops between the fast flowing upper part of the flow and the low-momentum lower part of the flow (Simpson 1989). BFS flows are characterized by a recirculating region between the step and reattachment point with negative horizontal velocities near the bottom. Another secondary recirculation region occurs in the immediate vicinity of the step corner. The mean reattachment point occurs where the streamline originating at the step edge impinges on the bottom wall. The statistical properties of turbulence for BFS flows have been studied extensively over the past two decades (Armali et al. 1983; Nakagawa and Nezu 1987; Simpson 1989; Chun and Sung 1998) and continue to be the subject of active experimental and numerical studies (Scarano et al. 1999; Spazzini et al. 2001; Kostas 2002; Piirto et al. 2003; Dejoan and Leschziner 2004; Furuichi et al. 2004; Nezu 2005; Schram et al. 2004; Sheu and Rani 2006).

The fundamental characteristics of the mean flow properties as a function of forest LAI will be explored using LES and the analysis focuses on two inter-related questions concerning possible recirculation zones near the edge and inside the forest canopy:

(1) To what extent does the flow near the forest edge into the clearing increasingly share important attributes with BFS flows (including the recirculation zone for the mean streamlines) as the forest LAI increases?

(2) Under what conditions does a recirculation zone set up downstream of the clearing inside the tall forest canopy. Can the formation of this recirculation zone be primarily described by inviscid flow calculations, at a minimum retaining the interplay between the mean pressure gradients (forcing the flow) and the drag force (opposing the flow)? 
It should be noted that these two recirculation zones have not been observed in previous LES studies. To explore these two questions, detailed simulations were conducted for a forest-clearing configuration that resembles the experimental set-up in Detto et al. (2007) for neutral atmospheric stability with the exception that the forest LAI progressively increases in simualtions. As discussed in Asner et al. (2003), terrestrial ecosystem LAI ranges from $1.3 \pm 0.9$ for deserts to $8.7 \pm 4.3$ for tree plantations, with temperate evergreen forests (needle-leaf and broadleaf) displaying the highest average LAI. Hence, the LES runs conducted here roughly span this entire range (2-8). To interpret the results, a simple analytical theory that predicts the onset of recirculation inside the canopy is proposed and tested against the LES results. Furthermore, simplified scaling arguments that decompose the mean velocity adjacent to the edge, but within the clearing, into a superposition of 'exit-flow' and BFS-like flow with their relative importance determined by the forest LAI are also explored.

\section{The Large-eddy Simulation}

Currently, LES is the state-of-the-art computational tool for investigating the structure of turbulence in a wide variety of settings, including plant canopies. Its use for modelling the atmospheric boundary layer dates back to the pioneering work of Deardorff (1972). Since then, developments in LES have proliferated (e.g., Moeng 1984; Schmidt and Schumann 1989; Mason and Thomson 1992; Sullivan et al. 1996; Kosovic 1997; Porte-Agel et al. 2000).

The use of LES as a tool to investigate turbulent flows inside and above forested canopies has rapidly expanded since the seminal work of Shaw and Schumann (1992) as evidenced by the large-number of studies thus far (e.g. Dwyer et al. 1997; Su et al. 1998, 2000; Shen and Leclerc 1998; Patton et al. 2001; Albertson et al. 2001; Yang et al. 2006a, b).

\subsection{The Equations}

A modified version of the LES code described in Albertson (1996) and Albertson and Parlange $(1999 \mathrm{a}, \mathrm{b})$ is used. The code solves the filtered Navier-Stokes equations written in rotational form (Orszag and Pao 1974) as,

$$
\frac{\partial \bar{u}_{i}}{\partial t}+\bar{u}_{j}\left(\frac{\partial \bar{u}_{i}}{\partial x_{j}}-\frac{\partial \bar{u}_{j}}{\partial x_{i}}\right)=-\frac{\partial p_{c}}{\partial x_{i}}-\frac{\partial \bar{\pi}}{\partial x_{i}}-\frac{\partial \tau_{i j}}{\partial x_{j}}+F_{i},
$$

where $t$ is time, $\bar{u}_{i}$ is the filtered velocity in direction $x_{i}$ and the total velocity is $u_{i}=\bar{u}_{i}+u_{i}^{\prime \prime}$ with $u_{i}^{\prime \prime}$ representing the subgrid-scale (SGS) or unresolved velocity. The $\tau_{i j}$ is the trace-free SGS stress defined as,

$$
\tau_{i j}=\left(\overline{u_{i} u_{j}}-\bar{u}_{i} \bar{u}_{j}\right)-\frac{\delta_{i j}}{3}\left(\overline{u_{k} u_{k}}-\bar{u}_{k} \bar{u}_{k}\right)
$$

The first term on the right-hand side of Eq. 1 is an imposed large-scale pressure gradient, which is uniform throughout the domain and is aligned in the streamwise direction (i.e. $\partial p_{c} / \partial x_{i}=0$ for $i=2,3$ ) and drives the flow. The total pressure per unit density, $p$, is therefore decomposed as, $p=p_{c}+\bar{p}+p^{\prime \prime}$. The quantity $\bar{\pi}$ is the modified resolved pressure defined as

$$
\bar{\pi}=\bar{p}+\frac{\delta_{i j}}{3}\left(\overline{u_{k} u_{k}}-\bar{u}_{k} \bar{u}_{k}\right)+\frac{1}{2} \bar{u}_{i} \bar{u}_{i},
$$


which is obtained to ensure that the continuity $\left(\partial \bar{u}_{i} / \partial x_{i}=0\right)$ is satisfied throughout the flow. To recover the true pressure, the SGS kinetic energy $q=\left(\overline{u_{k} u_{k}}-\bar{u}_{k} \bar{u}_{k}\right) / 2$ is required, but is not explicitly solved here; instead it is estimated following the standard dynamic approach (Germano 1992; Germano et al. 1991) as suggested by Sagaut (2002, p. 112, see also Xie et al. 2004), where $q=\left(\bar{u}_{i}-\widetilde{\bar{u}}_{i}\right) / 2$; the tilde $\sim$ represents a test filter and is usually taken to be twice the scale of the grid. The trapezoidal integration rule discussed in Gullbrand and Chow (2003) is used for this purpose.

The SGS stress is parameterized using a simple Smagorinsky (1963) model

$$
\tau_{i j}=-2 v_{T} \bar{S}_{i j}
$$

where $\bar{S}_{i j}=\left(\partial \bar{u}_{i} / \partial x_{j}+\partial \bar{u}_{j} / \partial x_{i}\right) / 2$ is the resolved strain-rate tensor and $v_{T}$ is the standard eddy viscosity given by $v_{T}=\left(C_{S} \Delta\right)^{2}|\bar{S}|,|\bar{S}|=\left(2 \bar{S}_{i j} \bar{S}_{i j}\right)^{1 / 2}$ is the magnitude of the resolved strain-rate tensor, and $C_{S}$ is the Smagorinsky constant known to depend on several factors (see e.g. Scotti et al. 1993; Sagaut 2002). In simulations of homogeneous turbulence, the predicted theoretical value is $C_{S} \approx 0.18$ but this value is known to be large when simulating boundary-layer flows and a value as low as $C_{S}=0.1$ has been used (see e.g. Lesieur et al. 2005). An intermediate $C_{S}=0.14$ is used here. The $\Delta=\left(\Delta_{x} \Delta_{y} \Delta_{z}\right)^{1 / 3}$ is the filter width following Deardorff (1970). The Smagorinsky model approach neglects, at the SGS level, the short-circuiting of the energy cascade in the inertial subrange due to the presence of the canopy. Other models can be used to account for this effect with an extra term in the equation for the SGS turbulent kinetic energy (e.g. Patton et al. 1998; Shaw and Patton 2003).

For consistency with the literature, index and meteorological notations are used interchangeably noting that streamwise, spanwise and vertical directions respectively, are $i(=1,2,3)$, or $(x, y, z)$. Following Shaw and Schumann (1992), the drag-force term is modelled as

$$
F_{i}=-C_{d} a|\overline{\mathbf{u}}| u_{i},
$$

where $a(x, y, z)$ is local leaf area density, i.e. area of plant surface for unit volume of space and $C_{d}(=0.2)$ is an isotropic drag coefficient (here taken from Katul and Albertson 1998). Note that $L A I(x, y)=\int_{0}^{h} a(x, y, z) d z$, where $h$ is the canopy height.

In these equations, the Coriolis force is neglected. This assumption was used in several LES studies for flow over and within a forested canopy (see e.g. Shaw and Schumann 1992; Yang et al. 2006a, b; Watanabe 2004; Dwyer et al. 1997). However, as pointed out by Wilson and Flesch (1999), the Coriolis force can be significant when modelling mean wind directions deep inside the canopy. Neutral atmospheric stability is assumed, thus avoiding the need to specify a heat forcing term (i.e. the gravitational term in the vertical momentum equation is neglected).

\subsection{Numerical Methods and Specification}

Equation 1 is solved on two nested computational staggered grids; the first one has a resolution $N_{x} \times N_{y} \times N_{z}$ on a domain $L_{x} \times L_{y} \times L_{z}$ that covers what can be considered a neutrally stratified boundary layer; the second grid covers a domain close to the surface $L_{x} \times L_{y} \times l_{z}$ that is only a small vertical fraction of the boundary layer (i.e. $l_{z}=\beta L_{z}$ with $\beta<<1$ ) and has an effectively finer resolution $n_{x} \times n_{y} \times n_{z}$.

The solution on each grid uses a pseudo-spectral method (Moin et al. 1978) with spectral differentiation in the two horizontal directions and finite difference in the vertical direction. A full de-aliasing is used in the spectral domain (see e.g. Canuto et al. 1988). As common in pseudo-spectral methods, the continuity equation is used to derive a Poisson equation for the 
modified pressure $\bar{\pi}$ that is then solved through a two-dimensional Fourier transform in the horizontal plane and a tri-diagonal matrix algorithm in the vertical direction (see e.g. Orlandi 2000). The time advancement uses a second-order explicit Adam-Bashforth scheme (see e.g. Orlandi 2000; Canuto et al. 1988).

Following the approach discussed in Khanna and Brasseur (1997) and Sullivan et al. (1996), the first grid communicates by a one-way coupling with the second grid hence simply giving initial and top-boundary conditions to the refined grid. This procedure allows for a higher resolution both vertically and horizontally without an excessive increase in the memory requirement and computational time since the two simulations are run in parallel. This two-grid strategy allows us to satisfactory resolve the canopy and the flow immediately above the canopy without the need of imposing artificial boundary conditions at the top of the small domain of interest. We note for example that in other simulations (e.g. Shaw and Schumann 1992; Dwyer et al. 1997; Patton et al. 2001) a frictionless rigid lid was imposed not far above the canopy, thus resulting in steeper vertical gradients of stress than those expected in the atmospheric boundary layer. More recently, Yang et al. (2006a) specified the forcing of the flow through the upper one-sixth of their computational domain (extending for six canopy heights). This second approach solves the problem generated by the rigid lid but, close to the forcing layer, the mean velocity profile is strongly altered with respect to the profiles observed in the atmospheric boundary layer (Yang et al. 2006a).

Periodic boundary conditions are used in the horizontal directions and zero vertical velocity is imposed at the ground surface. Here, the stresses are computed from a prescribed roughness length and the resolved horizontal velocity at one-half grid spacing above the surface (see Albertson and Parlange 1999a, b), an approach that is commonly used in LES of the planetary boundary layer (e.g., Moeng 1984; Schmidt and Schumann 1989). The external grid uses a frictionless rigid lid at the upper boundary.

The coarse-grid simulations extend for $500 \mathrm{~m}$ vertically and about $1,050 \mathrm{~m} \times 1,050 \mathrm{~m}$ horizontally while the fine-grid simulations extend $75 \mathrm{~m}$ vertically. A $20 \mathrm{~m}$ tall forest covers homogeneously the first half of the horizontal domain in the downstream direction.

For the coarse-grid simulations, a grid of $64 \times 64 \times 121$ is applied and the forest has been simulated as a distributed drag force extending vertically for $32 \times 64 \times 5$ nodes. This configuration gives an aspect ratio between the horizontal and vertical spacings $\left(\Delta_{x} / \Delta_{z}, \Delta_{y} / \Delta_{z}\right)$ of about 4. This aspect ratio should not introduce significant deviations from the isotropic grid solution (Scotti et al. 1993; Kaltenbach 1997). However, this point was investigated by running a simulation with a grid of $128 \times 128 \times 121$ and no overall sensitivity to the grid size, at the elevations where the boundary conditions are transferred to the more resolved grid, was found.

For the fine-grid simulations, a $128 \times 128 \times 44$ grid was used and the forest was introduced as a distributed drag force extending for $64 \times 128 \times 12$ grid nodes. With this configuration, the aspect ratio between the horizontal and vertical cell size in the fine grid is about 4.7. Although this is acceptable, we further investigated possible effects of the grid anisotropy and size using a simulation with a resolution of $256 \times 256 \times 44$ and corresponding aspect ratio below 2.4. These simulations did not reveal any significant differences in the statistics relevant to the study objectives.

The roughness length of the grass clearing was set at $z_{0}=0.1 \mathrm{~m}$ and is significant only in the fraction of the domain not covered by the canopy; otherwise the important sink of momentum is in the upper layers of the canopy and it is explicitly resolved by the drag force. 
Table 1 Basic characteristics of the simulated canopies

\begin{tabular}{lllll}
\hline Experiment & Vertical shape & Inflow edge (extension) & Outflow edge & LAI \\
\hline 1 & Forest & Smooth $(0.4 h)$ & Sharp & 2 \\
2 & Forest & Smooth $(0.8 h)$ & Sharp & 4 \\
3 & Forest & Smooth $(1.2 h)$ & Sharp & 6 \\
4 & Forest & Smooth $(1.6 h)$ & Sharp & 8 \\
5 & Constant & Smooth $(1.6 h)$ & Sharp & 8 \\
6 & Constant & Sharp & Sharp & 8 \\
\hline
\end{tabular}

The resolution in all simulations was $\Delta_{x}=\Delta_{y} \cong 0.4 h, \Delta_{z} \cong 0.08 h$

\section{Numerical Experiments}

The forest configuration reported above resembles the Duke Forest set-up (e.g. Ellsworth et al. 1995; Katul and Albertson 1998; Albertson et al. 2001). However, we emphasise that LAI will be varying across simulations and no attempt is made to reproduce the same forest density $a(x, y, x)$ reported in Katul and Albertson (1998). The basic configurations of the numerical experiments are reported in Table 1. The canopy shape refers to the vertical distribution of the canopy leaf area density and the dimensionless forest leaf area density reported in Fig. 1 is scaled with the LAI to have a unit integral. Table 1 reports the type of horizontal transition between the free air and the canopy. Most simulations here used a smooth transition at the grass-to-forest transition - gradually increasing from $\mathrm{LAI}=1$ towards the highest density level over a distance listed in parentheses in Table 1. This transition minimizes the Gibbs instabilities. The singular difference between the smooth transition and the sharp one is an

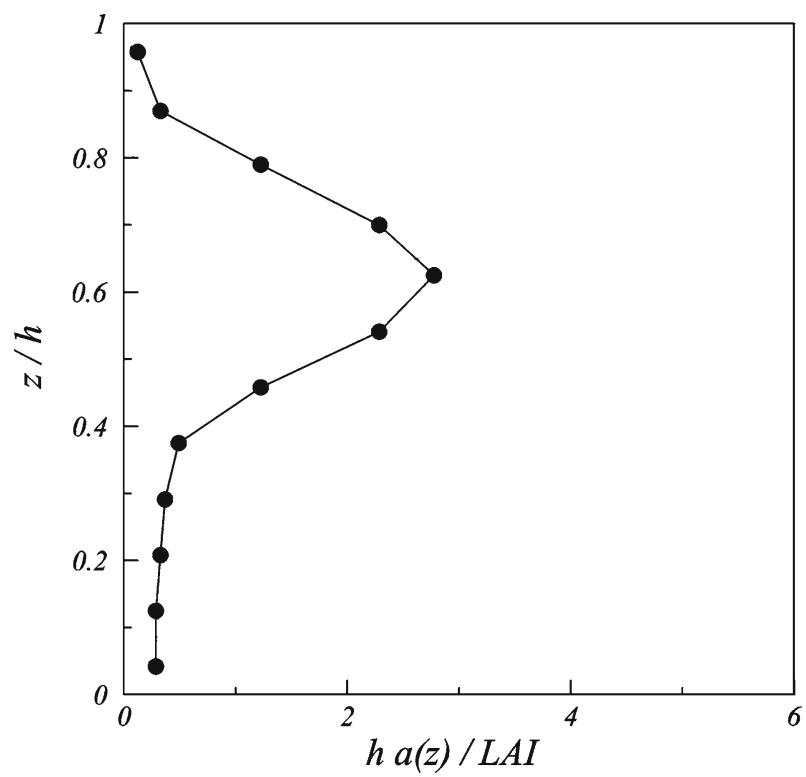

Fig. 1 Variations of the normalized canopy leaf area density with height. Solid circles and continuous line represents the normalized leaf area density used in LES simulations 
obvious small downwind shift in the equilibrium profiles inside the canopy, and this effect will be fully discussed below.

The simulations reported in Table 1 use upper boundary and initial conditions from a larger domain simulation. Therefore, a short discussion of the results obtained from the larger domain using a coarse grid and an intermediate LAI $(=4)$ is first presented, followed by results from the fine-grid simulations in the canopy sublayer for progressively increasing LAI.

\subsection{Coarse-grid Simulation}

The two primary statistics obtained by averaging the velocity and shear stress over time and horizontal planes are displayed in Fig. 2. Hereafter, such averaging is represented by \langle\rangle$_{x y t}$. The turbulent field is not statistically homogeneous along the mean wind direction because of the clearing-forest transition; hence, averaging across horizontal planes is questionable. However, we expect that these statistics will not be completely different from those of a turbulent flow over a homogeneous canopy allowing a rough evaluation of the simulation.

Figure $2 \mathrm{a}$ shows the scaled mean velocity as function of the scaled vertical coordinate. Here $z_{i}$ is the boundary-layer depth and

$$
u_{s 1}=\left|\langle u(x, y, h+\Delta z, t) w(x, y, h+\Delta z, t)\rangle_{x y t}\right|^{1 / 2},
$$

is a velocity scale computed at the first level above the canopy height. It is evident that the presence of the drag in half of the domain retards the flow below the canopy top. Above the canopy, the mean flow tends to assume a logarithmic profile, and values above $z=0.9 z_{i}$ are not shown since a 'damping layer' is used to absorb fluctuations close to the lid (see e.g. Mason and Thomson 1987). Figure 2b shows the scaled mean correlation between $w^{\prime}$ and $u^{\prime}$ as a function of the scaled vertical coordinate. Here, primed quantities are fluctuations from the averaged state e.g. $w^{\prime}=w-\langle w\rangle_{x y t}$. Note that even for this coarse resolution, most of the stress is resolved. The total stress decays properly to zero at the top of the domain with a near constant slope. Figure 2c shows the scaled turbulent kinetic energy (TKE), defined here as $\left\langle u_{i}^{\prime} u_{i}^{\prime}\right\rangle_{x y t} / 2$. As for the stress, most of the TKE is resolved. Recall that the subgrid TKE has been evaluated dynamically. In short, the overall profiles from the coarse resolution resemble boundary-layer flows over a canopy.
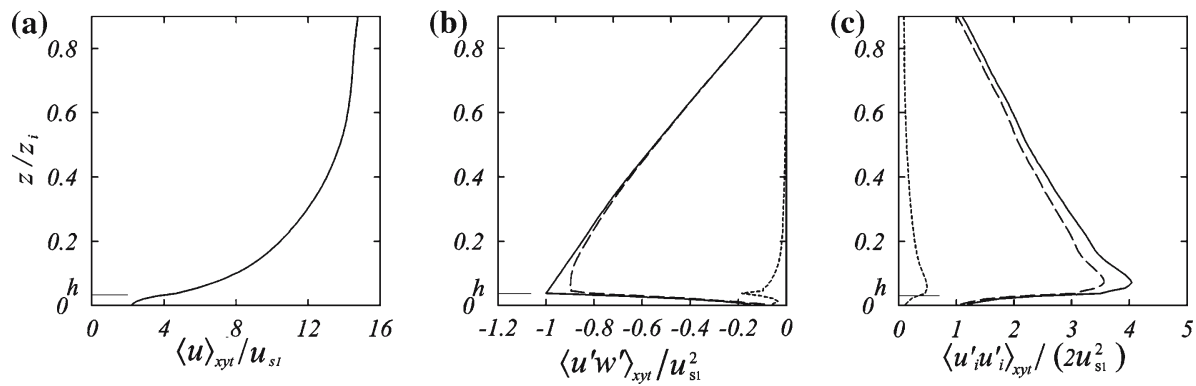

Fig. 2 Plane and time-averaged values of the mean streamwise velocity (a), turbulent stresses (b) and turbulent kinetic energy (c) as a function of normalized height. $z_{i}$ is the boundary-layer height. In (b) and (c) dashed lines are the resolved predictions, dotted lines the subgrid-scale components and continuous lines the total values. $u_{s} 1$ is a velocity scale defined in the text 

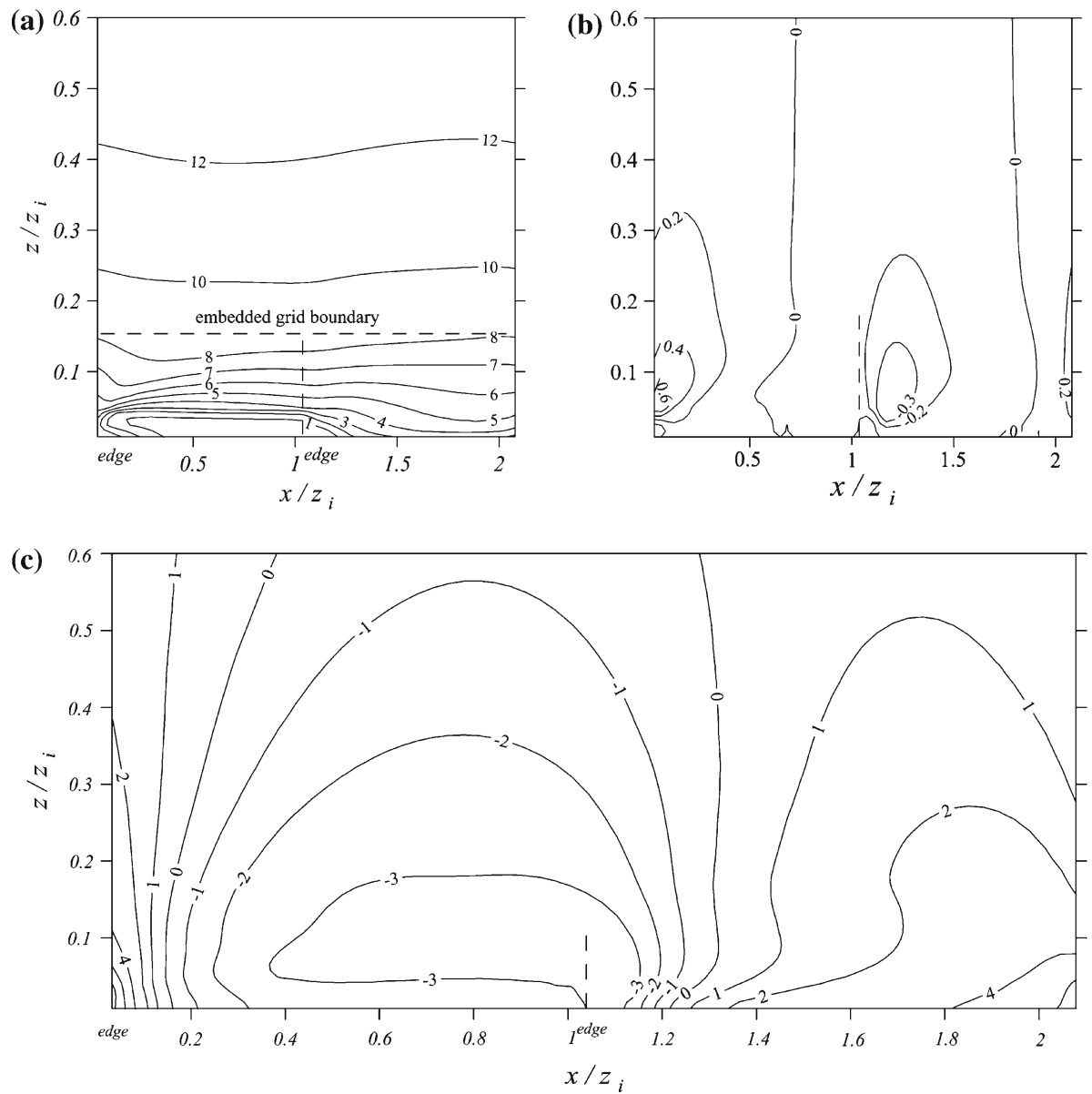

Fig. 3 Time and crosswind averaged values of: (a) mean horizontal velocity $\langle u\rangle_{y t} / u_{s 2}$; (b) mean vertical velocity $\langle\bar{w}\rangle_{y t} / u_{s 2} ;$ (c) pressure $\langle\bar{p}\rangle_{y t} / u_{s 2}^{2}$, as a function of the normalized height above the ground for experiment 2 (see Table 1). $z_{i}$ is the boundary-layer height. Edge indicates the horizontal location of the forest boundaries. In (a) the position of the boundary between coarse and refined simulation is indicated. $u_{s 2}$ is a velocity scale defined in the text

Figure 3 presents the time and crosswind averaged statistics, and hereafter, angled brackets \langle\rangle$_{y t}$ represent such averaging. Figure 3 a shows the mean velocity $\langle u\rangle_{y t}$ contours scaled with $u_{s 2}=\left|\left\langle u\left(x_{*}, y, h+\Delta z, t\right) w\left(x_{*}, y, h+\Delta z, t\right)\right\rangle_{y t}\right|^{1 / 2}$, with $x_{*}$ being the coordinate of the canopy-to-grass edge minus three canopy heights. The $\langle u\rangle_{y t}$ contours, as a function of elevation above ground and downwind distance from the clearing-to-forest canopy edge, are shown. The canopy $(L A I=4)$ can be identified on the left half of the figure while the clearing is on the right half. With this resolution and LAI, no mean recirculation is evident. The horizontal dashed line here shows the embedded grid boundaries (i.e. the position where the velocity is passed to the fine-grid simulation). Figure $3 \mathrm{~b}$ shows the mean vertical velocity $\langle w\rangle_{y t}$ contours scaled with $u_{s 2}$ plotted as a function of elevation above ground and downwind distance from the clearing-to-forest canopy edge. Note the positive mean velocity at the clearing-to-forest edge and the negative value at the forest-to-clearing edge. For reference, 

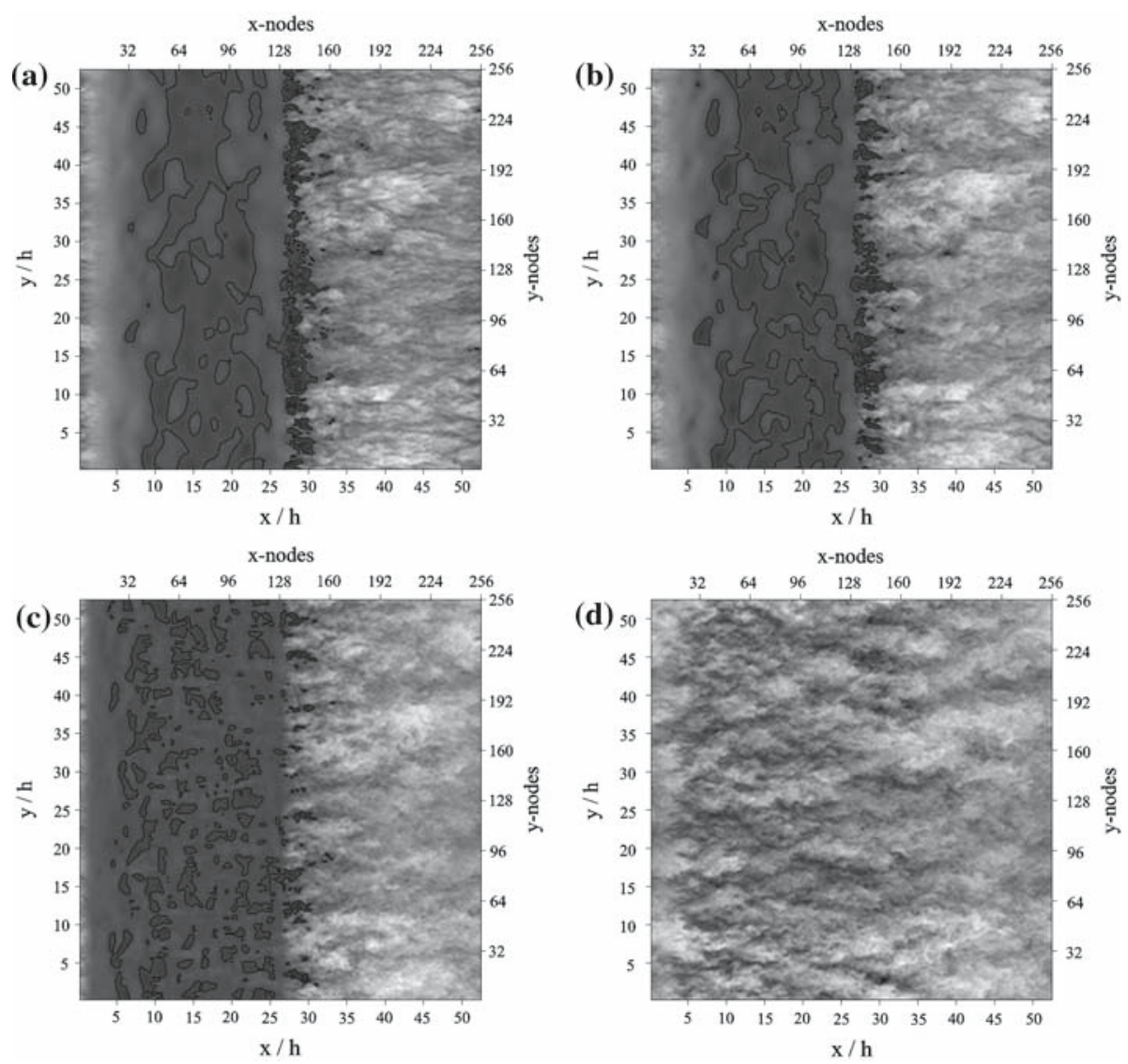

Fig. 4 Variations of the instantaneous velocity in the streamwise direction $(\bar{u})$ across horizontal sections at four elevations above ground for $\mathrm{LAI}=8$ and resolution $256 \times 256$. Node 128 (on the $x$ axes) marks the forest-to-clearing edge. Dark and bright grey correspond to lower and higher velocities, respectively. Areas of negative velocities are bounded by a continuous line. (a) close to the ground; (b) Fourth node above the ground, corresponding to $h / 3$; (c) Eighth node above the ground, corresponding to $2 h / 3$; (d) Sixteenth nodes above the ground, corresponding to $4 h / 3$

Fig. $3 \mathrm{c}$ shows the variations in the mean pressure field scaled with $u_{s 2}^{2}$ and plotted as the variation from its averaged value over the simulation domain. Recall that in this case, only gradients are important.

\subsection{Fine-resolution Simulations}

The main objective of this work is to investigate the singular effects of canopy LAI on the mean flow across forest edges focusing on two regions - the flow inside the forest canopy and the flow near the outflow forest edge. Before starting a quantitative analysis on averaged quantities, a qualitative picture of the instantaneous velocity field is presented. Figure 4 shows the snapshots of the instantaneous streamwise velocity at four different elevations above the ground surface using simulations with the maximum LAI (=8). For this qualitative analysis, the horizontal resolution of $256 \times 256$ is used. Figure $4 \mathrm{a}-\mathrm{c}$ represent the flow below 

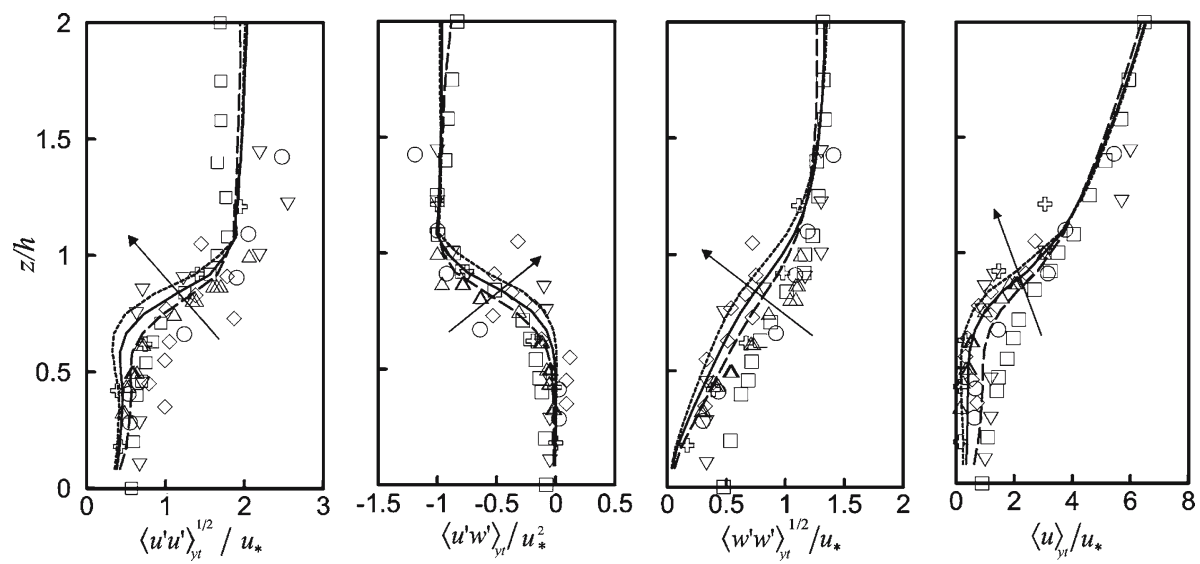

Fig. 5 Comparison between statistics from LES runs ( $\mathrm{LAI}=2$, 4, and 8, see Table 1) and various experiments with different canopy densities: Duke pine forest (circle), corn (triangle), hardwood forest (inverse triangle), rice (diamonds), rods (squares) and spruce forest (crosses). Results are plotted as a function of the normalized elevation where $h$ is the canopy height. Direction of the arrow indicates an increase in LAI for the simulations

the canopy top while $4 \mathrm{~d}$ shows the flow above the forest. Modelled canopy drag in the first half of the domain (up to 128 nodes in the $x$ direction) reveals the expected slow down of the flow, but more important is the complete shift in turbulence "texture" as the flow traverses the forest edge into the clearing. Inside the canopy, several areas of weak negative instantaneous velocity can be recognized as coherent regions bounded by continuous black lines. Increasing the elevation above the ground decreases the total area covered by negative velocity. Similar areas of negative velocity are present for lower LAI (2-6) (not shown here) but the total area of coverage decreases with decreasing LAI. The effects of this variation on the mean flow characteristic will be explored in detail later. More intense negative velocities are observed after the forest-to-clearing edge. Here the negative velocities appear to resemble BFS-like effects with a separation and mean recirculation (e.g. Nakagawa and Nezu 1987; Simpson 1989) and, as before, the total area covered by negative velocity decreases with decreasing LAI. As expected, the areas with negative velocity tend to disappear near the forest top. However, the presence of the canopy drag can be seen since the turbulence "texture" remains completely different above the canopy and above the clearing (Fig. 4d).

Figure 5 compares the flow statistics reported in several field and laboratory experiments with the flow statistics computed by the LES here at location $x_{*}$. These measurements span a wide range of LAI values (2-6) and $h(0.12-25 \mathrm{~m})$, and include a corn canopy (Wilson et al. 1982), a hardwood forest (Meyers and Baldocchi 1991), a rice canopy (Katul et al. 2001), a Spruce forest (Amiro 1990), a pine plantation (Katul and Chang 1999), and densely arrayed steel rods in a flume (Poggi et al. 2004). Complete details of these experiments can be found in Katul et al. (2004) and will not be repeated here. The field measurements are scaled with the local measured $u_{*}$ and canopy height while the LES results have been scaled with $u_{s 2}$, representing here the local $u_{*}$. The LES simulations agree with the observed flow statistics lending confidence to the realism of these simulations.

Figure 6 shows the spatial variation of the horizontal mean velocity (scaled with $u_{s 2}$ ) as LAI increases (see Table 1, first four cases). With an LAI $=2$, no recirculation zones are observed in agreement with previous LES results reported by Yang et al. (2006a) despite differences in canopy density distribution, forcing method, and subgrid-scale parameterization. 

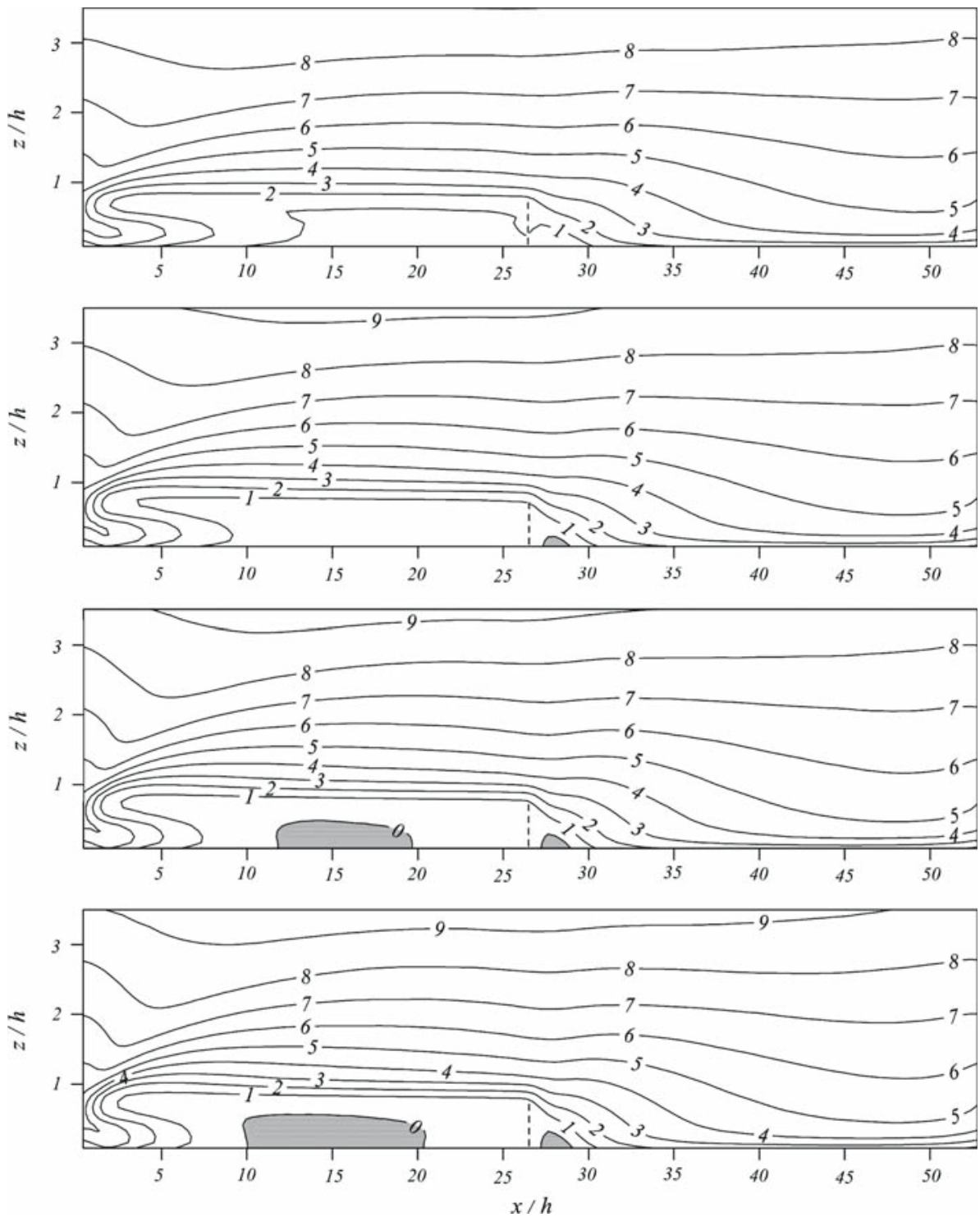

Fig. 6 Contours of the mean streamwise velocity $\langle u\rangle_{y t}$ scaled with $u_{s} 2$ for different LAI values. Top to bottom: experiment 1, 2, 3, 4 (see Table $1 ; \mathrm{LAI}=2,4,6,8)$. Grey areas correspond to negative velocity; $h$ is the canopy height

With this value of LAI, it appears that the 'exit flow' model is a good approximation for the outflow at the forest-to-clearing edge. In the second panel $(\mathrm{LAI}=4)$, a small recirculation zone is observed at the outflow edge, showing that the 'exit flow' approximation is no longer reasonable because the recirculation structures become a dominant contributor to the average velocity at this LAI (see also Fig. 4). At the inflow edge the contours of the mean horizontal velocity at $\mathrm{LAI}=4$ are similar to the ones simulated for $\mathrm{LAI}=2$, but the isolines are closer to each other suggesting a stronger decrease in mean velocity over a comparable distance. 
A further increase in LAI $(=6)$ completely changes the behaviour of the flow inside the canopy. A weak recirculation zone can be observed extending for about $8 h$ and characterized by a width much larger than the mixing length for canopy turbulence. The behaviour at the outflow edge is similar to the one at $\mathrm{LAI}=4$, although an increase in the recirculation area and intensity can be noted. Increasing the LAI to 8 does not significantly change the overall patterns. However, the recirculation inside the canopy increases its extension. At the forest-to clearing edge, a small increase in the recirculation area is noted suggesting an asymptotic limit is approached.

The results in Fig. 6 have been obtained with the leaf area density profile reported in Fig. 1 and a smooth transition in the streamwise direction at the clearing-to-forest edge; this is somewhat arbitrary. To further explore the influence of these two factors on the mean flow and in particular on the recirculation inside the canopy we performed two simulations with $L A I=8$; experiments 5 and 6 in Table 1. The results are reported in Fig. 7 along with the standard configuration result for reference. The results from a constant density but smooth transition simulation show completely different velocity isolines at the clearing-toforest edge. In particular, we observe the disappearance of the so-called low-level jet (Wilson and Flesch 1999; Li et al. 1990) associated with the vertical profile of the leaf area density (i.e. the small leaf area density close to the ground generated higher wind speed and with a constant leaf area density profile this feature disappeared). However, inside the canopy, the recirculation zone retains a similar extension while appearing closer to the clearing-to-forest edge. This is expected because the constant leaf area density profile has more foliage near the ground and exerts more drag on the horizontal mean velocity in this zone. Eliminating the smooth transition, Fig. 7c shows no major changes for the forest canopy flow except a minor shift towards the clearing-to-forest boundary for the onset of recirculation. As expected no visible changes are observed at the forest-to-clearing edge. Hence, from this sensitivity analysis, the onset of a recirculation inside the canopy is weakly dependent on the precise characteristic of the transition and mainly depends on LAI values.

To further evaluate our simulations, we compare the simulation results at the two edges with experiments and RANS simulations reported elsewhere. The published RANS simulations of Wilson and Flesch (1999) for the experiments by Raynor (1971) and Flesch and Wilson (1999) provide the necessary data and RANS model output. We did not attempt to reproduce these experiments and simulations precisely. However, comparison is possible because one of the leaf area density profiles used in our LES simulations is similar to that used by Wilson and Flesch (1999). We selected, among our simulations, the LES runs with $C_{d} L A I$ values closest to those of Wilson and Flesch (1999). Figure 8, left, shows the comparison with the simulations and experiments at the clearing-to-forest transition. The vertical profiles refer to different distances from the edge. All the results were scaled with the respective mean velocity at $x \approx 6 h, z=2 h$. In Wilson and Flesch (1999), the Coriolis force was considered; therefore, the magnitude of the horizontal velocity vector is shown. Despite these differences and other minor differences in leaf area density profiles (our profile is less dense near the ground and more dense in the vicinity of $z=(3 / 4) h)$, reasonable agreement is observed with both the RANS model calculations and the experiments of Raynor (1971). In particular, the low-level jet noted in the RANS model calculations and the measurements in Raynor (1971) was resolved by the LES here. Li et al. (1985) reported similar findings using RANS model calculations, but these results are not repeated. Figure 8 shows further comparisons with the simulations of Wilson and Flesch (1999) for the forest-to-clearing transition (right). Both simulations are scaled with the respective mean velocity at $z=h$ and one canopy height before the forest-to-clearing transition. Based on their reported $C_{d} a h$, our simulations conducted with an $\mathrm{LAI}=4$ should be of similar density. For reference, the LES results 

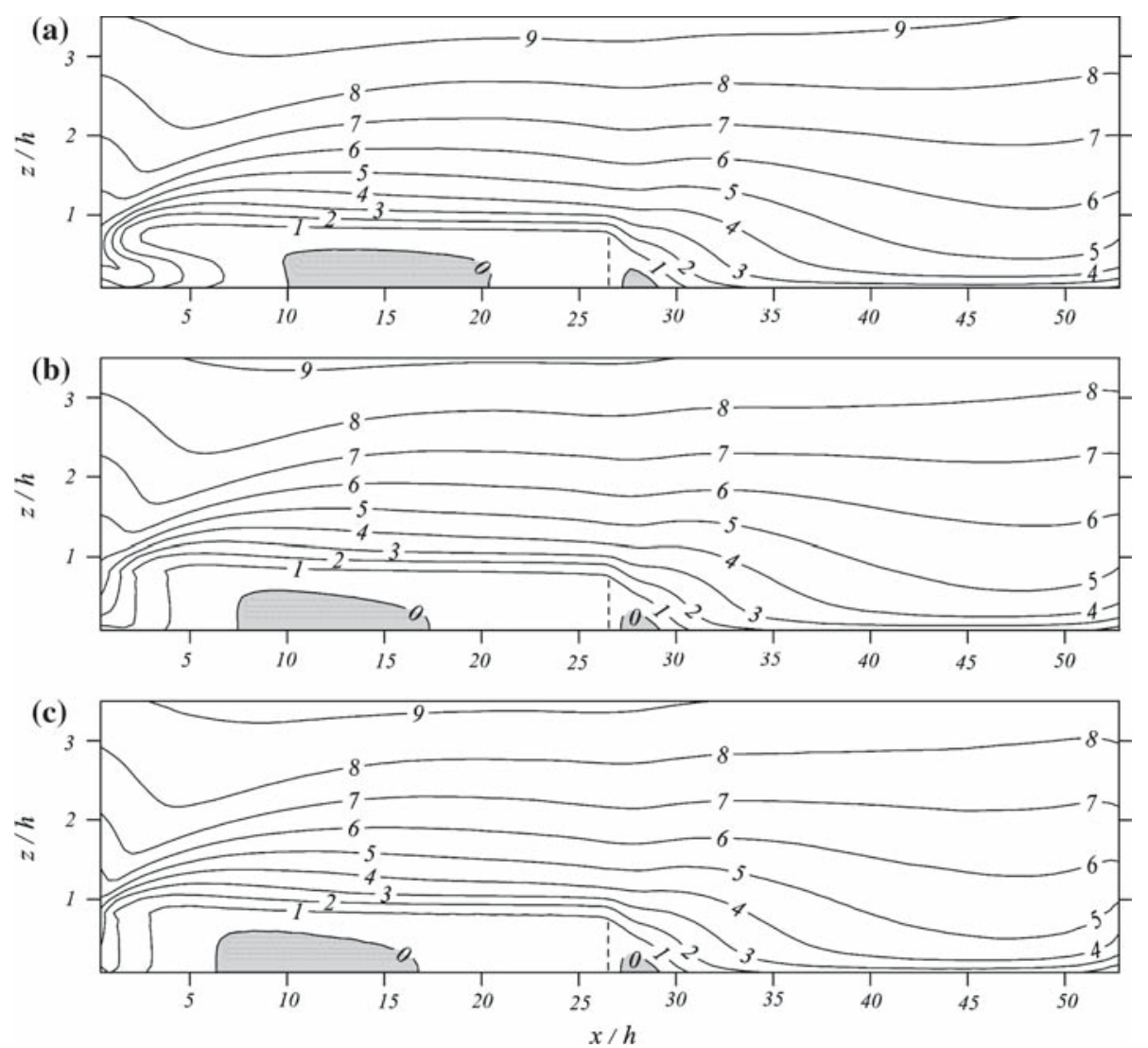

Fig. 7 Contours of the mean streamwise velocity $\langle u\rangle_{y t}$ scaled with $u_{s 2}$ and for LAI $=8$ and different clearing-to-forest edge conditions. (a) clearing-to-forest transition and forest type density (experiment 4); (b) smooth clearing-to-forest transition and vertically constant leaf area density (experiment 5) and (c) sharp clearing-to-forest transition and vertically constant leaf area density (experiment 6,). Grey areas correspond to negative velocities; $h$ is the canopy height

with LAI $=8$ are also included. The simulations here and the RANS model calculations in Wilson and Flesch (1999) agree on the existence of a recirculation zone below $z / h=0.2$. However, the LES simulations show a shorter mean recirculation zone when compared to the RANS model results in Wilson and Flesch (1999). Similar results (not reported here) were also obtained by Liu et al. (1996) using a $K-\omega$ RANS model, who noted a reversed mean velocity in the domain area bounded by $z=0.03 h$ to $0.15 h$, and $x=1 h$ to $2 h$. These authors also noted that recirculation was present for $\mathrm{LAI}=10$ but disappeared for $\mathrm{LAI}=4$. They argued that while the mean recirculation was visually observed in their experiment, the hot wire anemometers used were not able to register it. The presence of this recirculation will be further discussed in Sect. 5 .

Up to this point, much of the discussion has centred on the mean longitudinal velocity. Next, the effects of LAI on the mean vertical velocity are considered. In Fig. 9, a comparison between the mean vertical velocity, $\langle w\rangle_{y t}$, for LAI $=2$ and 8 , (experiments 1 and 4 ) is presented. The overall difference is not large but consistent with the preceding figures. At greater LAI the higher drag force significantly slows down the flow thus allowing the change in sign 

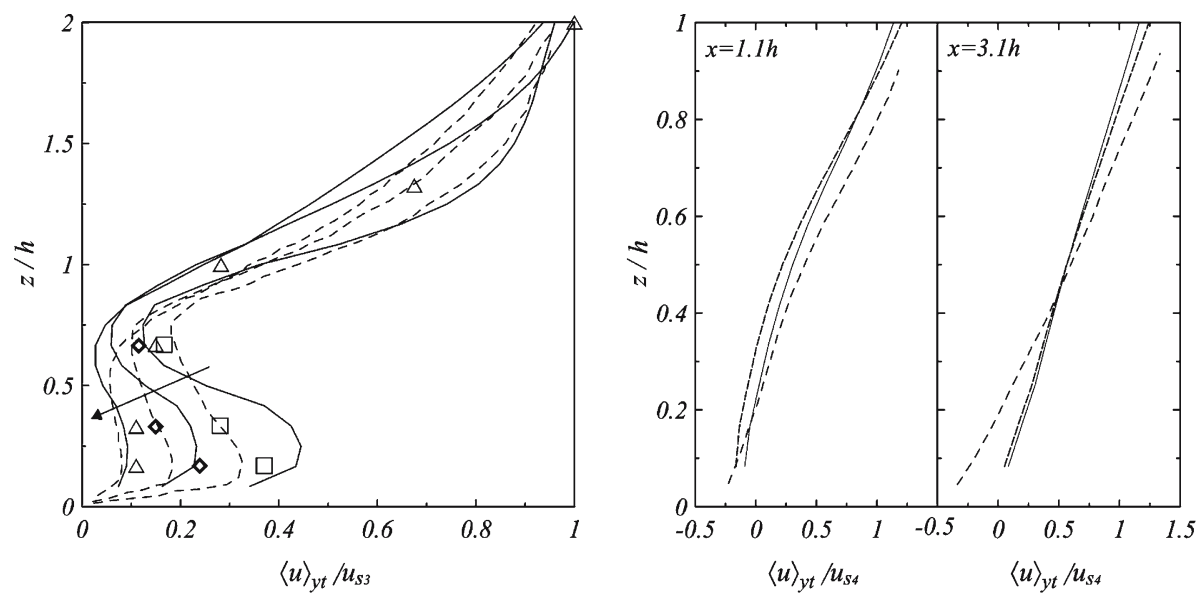

Fig. 8 Left panel: vertical profiles of mean streamwise velocity $\langle u\rangle_{y t}$ immediately after the clearing-to-forest edge. $u_{s 3}$ is the velocity at $x \approx 6 h, z=2 h$. Symbols: experiment of Raynor (1971). Open square, $x \approx 2 h$; open diamonds, $x \approx 4 h$; open triangle, $x \approx 6 h$. Dashed lines: RANS simulations of the same experiment by Wilson and Flesch (1999). Continuous lines: present simulations (experiment 4) LAI $=8$. The arrow shows the direction of increasing distance from the edge for the simulations. Right panel: vertical profiles of mean streamwise velocity $\langle u\rangle_{y t}$ immediately after the forest-to-clearing transition. $u_{s 4}$ is the velocity at $z=h$ and $x$ one canopy height before the forest-to-clearing transition. Long dashed line: RANS simulations of the Manning experiment by Wilson and Flesch (1999). Continuous line: present simulation with LAI =4. Short dashed lines: present simulations with $\mathrm{LAI}=8$
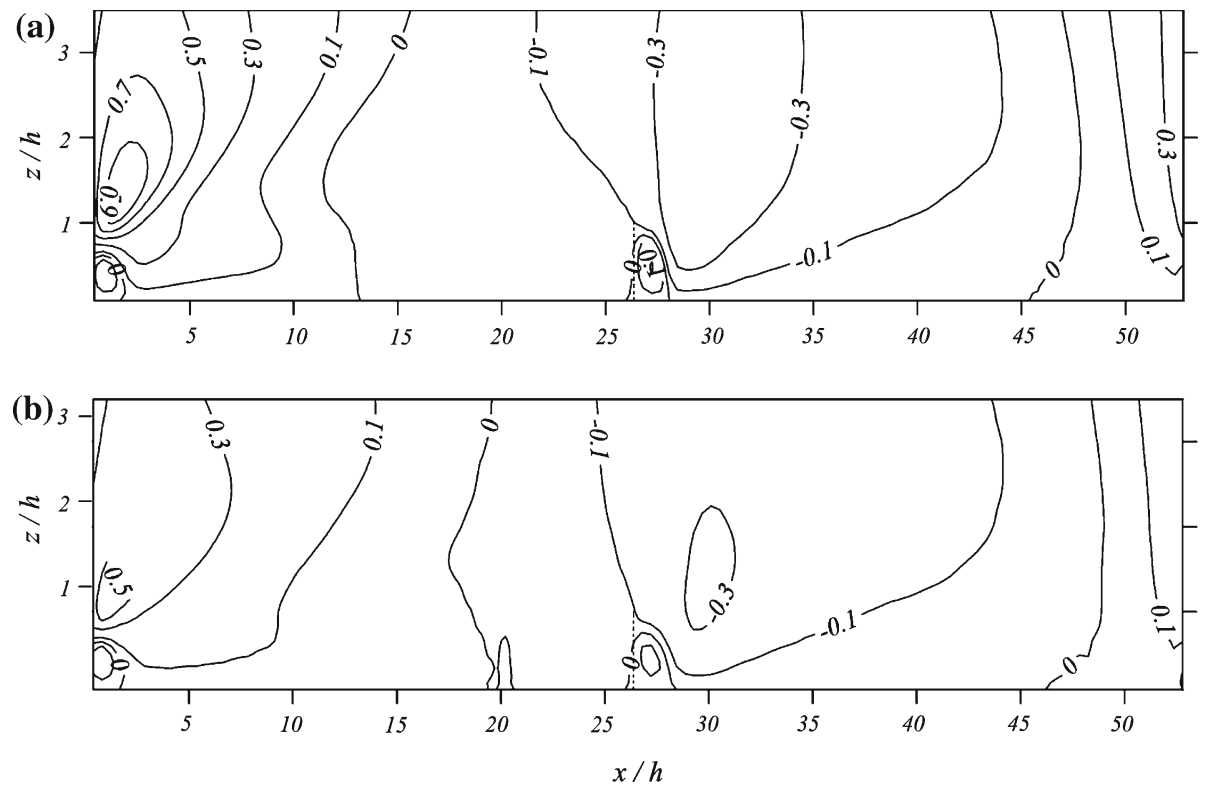

Fig. 9 Contours of mean vertical velocities $\langle\bar{w}\rangle_{y t}$ scaled with $u_{s 2}$, (a) LAI $=8$ and (b) LAI $=2$. Experiments 1 and $4 ; h$ is the canopy height 

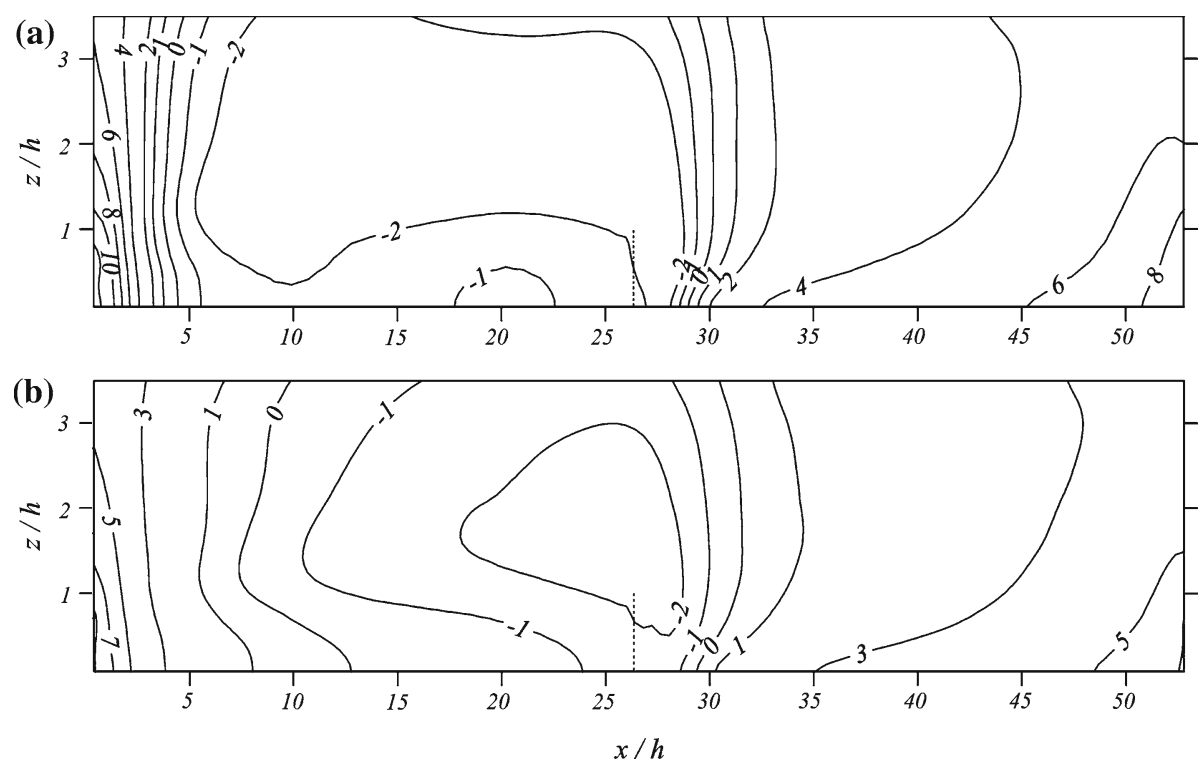

Fig. 10 Contours of mean pressure $\langle\bar{p}\rangle_{y t}$ scaled with $u_{s 2}^{2}$ and plotted as a variation from its volume-averaged value (see text); (a) $\mathrm{LAI}=8$ and (b) $\mathrm{LAI}=2$. See experiments 1 and $4 ; h$ is the canopy height

of the vertical velocity within the recirculation inside the canopy. A stronger positive velocity is observed for the greater LAI immediately at the forest-to-clearing boundary consistent with the onset of another recirculation zone.

More interesting is the mean pressure field reported in Fig. 10; this is scaled with $u_{s 2}^{2}$ and plotted as a departure from its averaged value over the simulation volume. For $\mathrm{LAI}=8$, the pressure field inside the canopy close to the ground shows a local maximum at $x / h=20$. The adverse pressure gradient between $10 h<x<20 h$ is consistent with the onset of a recirculation. This feature is completely absent in the simulation with $\mathrm{LAI}=2$ (no recirculation). No clear qualitative distinction can be observed in the pressure field after the outflow edge.

\subsection{Mean Momentum Balance for the Fine Grid}

The relative importance of the various terms in the time-averaged longitudinal mean momentum balance is explored next so as to isolate the key processes responsible for the onset of recirculation. The resolved-scale mean momentum equation can be written as (using $x, y, z$ coordinates):

$$
\begin{aligned}
& 0=-\langle u\rangle_{y t} \frac{\partial\langle\bar{u}\rangle_{y t}}{\partial x}-\langle\bar{w}\rangle_{y t} \frac{\partial\langle\bar{u}\rangle_{y t}}{\partial z}-\frac{\partial p_{c}}{\partial x}-\underset{\text { term } 2}{2} \underbrace{}_{\text {term } 3} \frac{\partial \bar{p}}{\partial x}\rangle_{y t}-\underset{\text { term } 4}{C_{d} a\langle|\overline{\mathbf{u}}| \bar{u}\rangle_{y t}} \\
& -\frac{\partial\left\langle\bar{u}^{\prime} \bar{u}^{\prime}\right\rangle_{y t}}{\partial x}-\left\langle\frac{\partial \tau_{x x}}{\partial x}\right\rangle_{y t}-\frac{\partial\left\langle\bar{u}^{\prime} \bar{w}^{\prime}\right\rangle_{y t}}{\partial z}-\left\langle\frac{\partial \tau_{x z}}{\partial z}\right\rangle_{y t} .
\end{aligned}
$$

As before, angle brackets denote an average over time and crosswind direction and the prime denotes a fluctuation of any variable from this average, e.g. $\bar{u}=\vec{u}+\langle u\rangle_{y t}$. Terms 1 and 2 represent the advection by the mean flow in the streamwise and vertical directions, 
respectively; term 3 represents the pressure gradient; term 4 is the canopy drag force; terms 5 and 6 are the turbulent transport from both resolved and subgrid scales in the streamwise and vertical directions, respectively.

The contours of each term for experiment 4 are presented in Fig. 11, with all values scaled with $u_{s 2}^{2} / h$. The sum of all the terms was verified to be near zero at all points in the spatial domain thereby lending confidence to the averaging intervals chosen here.

We start our analysis here by discussing the terms in the mean momentum balance immediately after the forest-to-clearing edge. Apart from the drag that is absent, all the terms have comparable magnitude and therefore none of them can be neglected in this region. This prevents any simplified analytical treatment in this zone. However, expanding the argument in Detto et al. (2007), we propose in Sect. 5 a simple superposition model to explain the mean flow dynamics in this area as a function of forest LAI. For small LAI, the flow resembles an exit flow, while for large LAI, the flow includes BFS-like signatures. For infinite LAI, the flow becomes identical to a standard BFS flow. Hence, for any intermediate LAI, it should be possible to compute the mean velocity as a weighted average of these two end-member states where the weights vary with LAI.

Inside the forest canopy, the pressure gradient and the drag force are the two dominating terms inside the recirculation area between $z<0.5 h$ and $10 h<x<20 h$ (Fig. 11). Changes in sign of the pressure gradient correspond to a change in the mean velocity within the recirculation area between $z<0.5 h$ and $10 h<x<20 h$, which also corresponds to an area of positive drag force.

All the terms for the mean momentum balance in the zone just before the onset of recirculation i.e. $0<x / h<10$ are presented in Fig. 12a. Advection in the vertical direction (term 2) and turbulent transport in the streamwise direction (term 5) are much smaller when compared to all the other terms in the balance and can be neglected. Based on this figure, a balance between pressure (term 3) and drag force (term 4) can be hypothesized near the ground $(z<0.1 h)$ across a horizontally integrated streamline for $0<x / h<10$. This balance is in agreement with the analysis of Holland (1989) for a deep forest. In this region, the mean longitudinal velocity can be approximated as a free shear and its vertical gradients can be neglected relative to the horizontal. Although the turbulent stress (term 6) and advection (term 1) can be comparable to the mean pressure gradient at some locations, their overall contribution to the mean momentum balance becomes negligible when horizontal averaging is applied in this region. Note that when the sign change is considered, neglecting terms 6 and 1 becomes even more reasonable because of cancellations.

\section{Scaling Analysis of the Recirculation Zone Inside the Forest Canopy}

\subsection{Basic Equations and Assumptions}

The time and crosswind-averaged Navier-Stokes equation is:

$$
U \frac{\partial U}{\partial x}+W \frac{\partial U}{\partial z}=-\frac{\partial P}{\partial x}-\left(\frac{\partial\left\langle u^{\prime} w^{\prime}\right\rangle_{y t}}{\partial z}+\frac{\partial\left\langle u^{\prime} u^{\prime}\right\rangle_{y t}}{\partial x}\right)+\left\langle F_{x}\right\rangle_{y t}
$$

For notational simplicity, defined $U=\langle u\rangle_{y t}$ and similarly for $W$ and $P$. As before, primes denote fluctuation from the time and crosswind averaged value i.e. $u=U+u^{\prime},\left\langle F_{x}\right\rangle_{y t}$ is the mean drag force, and the other bracket terms are the gradients of turbulent Reynolds stress and the longitudinal velocity variance, respectively. 


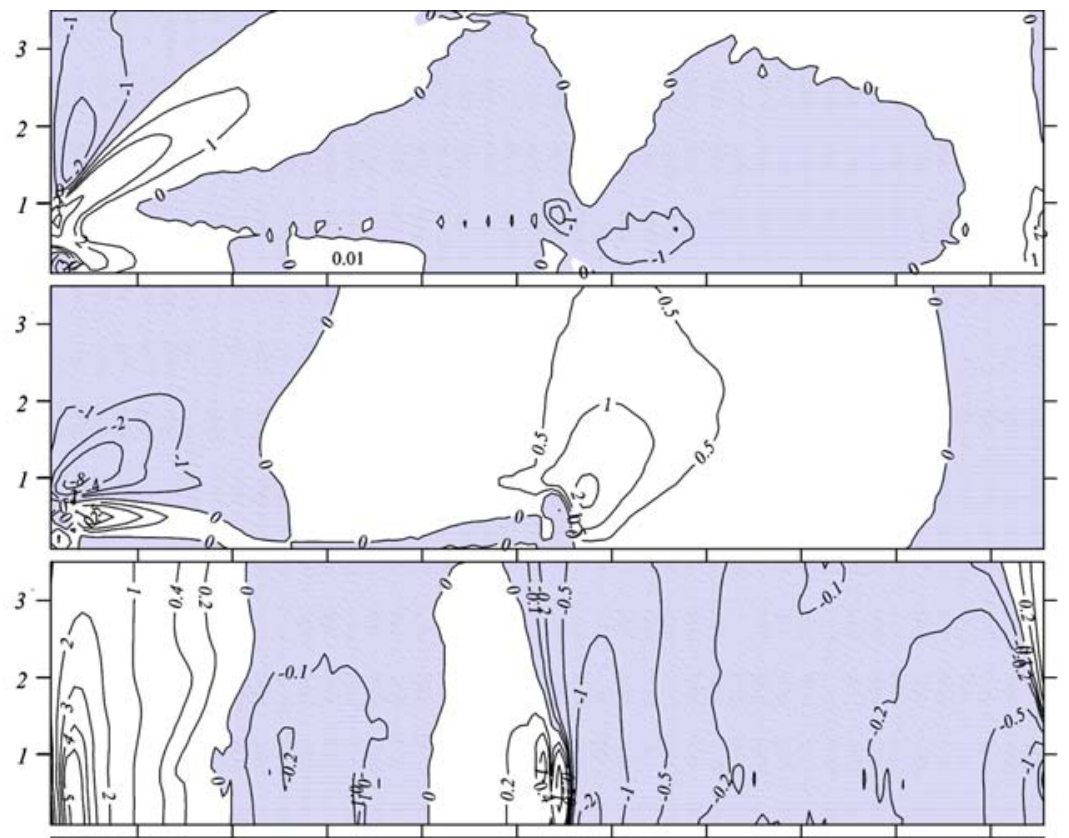

Term $I$

Term 2

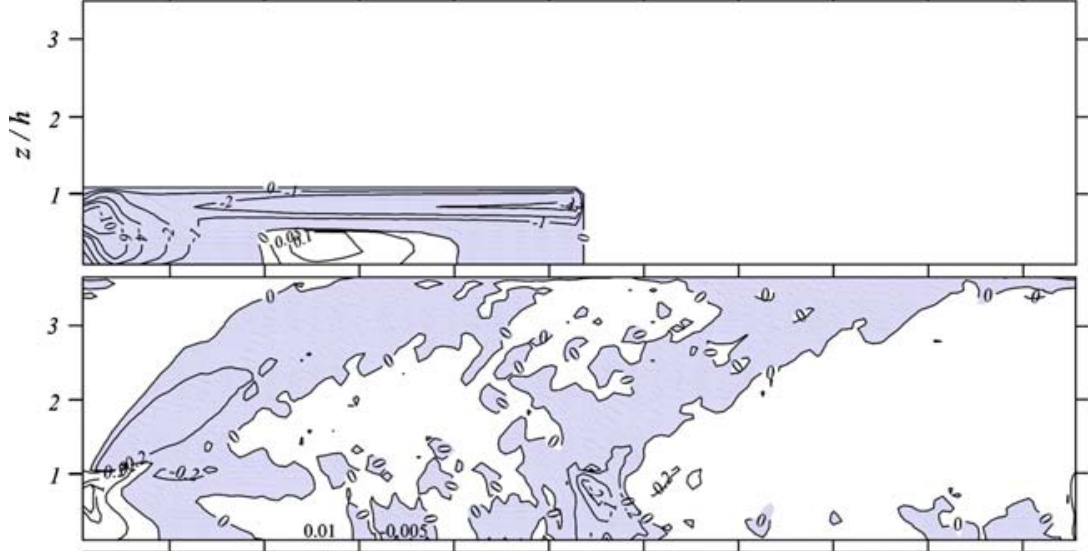

Term 4

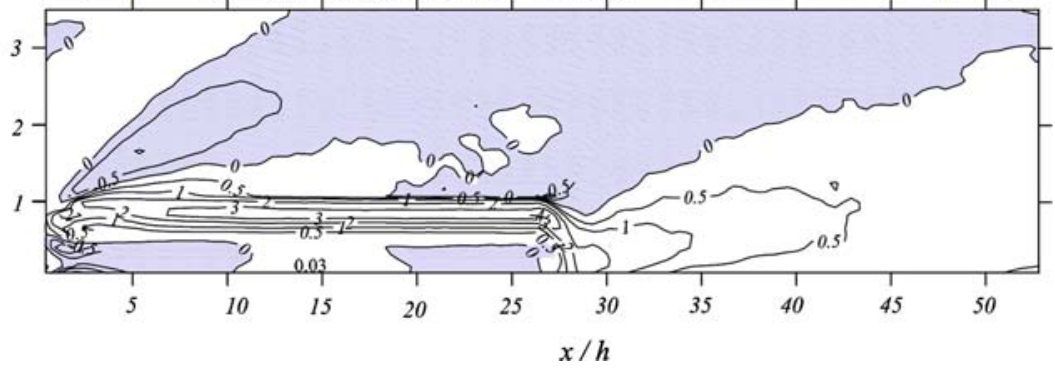

Term 5

Term 6

Fig. 11 The terms in the mean longitudinal momentum balance scaled with $h / u_{s 2}^{2} . h$ is the canopy height. Term 1 is advection in the streamwise direction; Term 2 is advection in the vertical direction; Term 3 is the pressure gradient force; Term 4 is the canopy drag force; Term 5 is the turbulent transport in the streamwise direction; and Term 6 is the turbulent transport in the vertical direction 


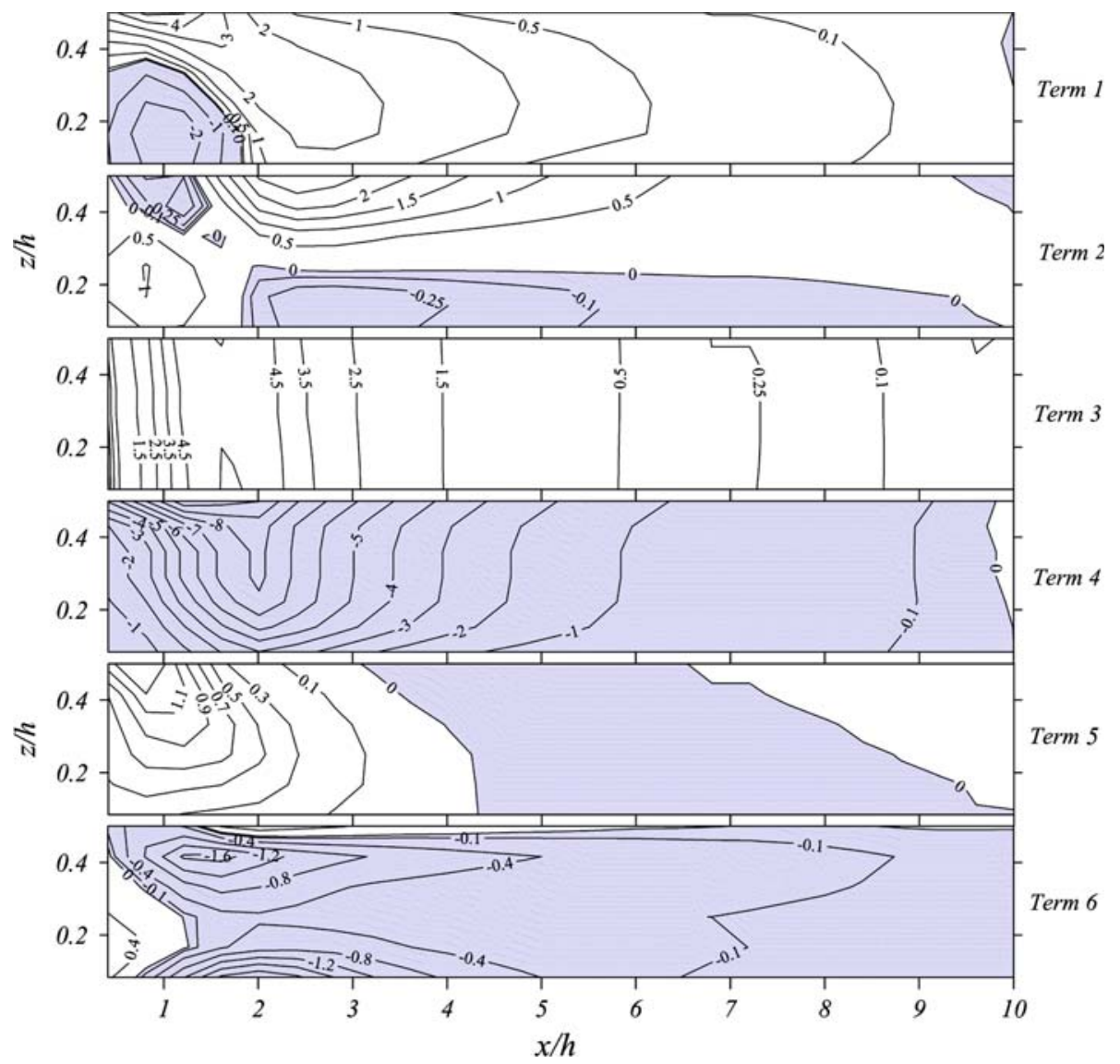

Fig. 12 Same as Fig. 11 but for a zoom in area just prior to the recirculation $(0<z / h<0.5 ; 0<x / h<10)$; $h$ is the canopy height

Following the analysis in the previous section, only two terms (drag and pressure gradient) dominate the mean momentum balance in the region near the ground within the forest canopy bounded between the entrance and recirculation zone. We explore next the onset of the longitudinal location of a recirculation zone from the entrance and explore how LAI modulates its occurrence.

\subsection{Onset of Recirculation in the Lower-canopy Levels}

The most simplified mean momentum balance in this region is given by

$$
C_{d} a|U| U=-\frac{\partial P}{\partial x}
$$

To determine the onset of recirculation, consider the integral momentum balance from $x=0$ (entrance) to $x=L_{s}$, the distance at which recirculation begins. The longitudinally integrated mean momentum balance yields 


$$
\int_{x=0}^{x=L_{s}} U^{2} d x=\frac{P(0)-P\left(L_{s}\right)}{C_{d} a}
$$

We arbitrarily assume that the mean velocity can be approximated by a power-law distribution with an unknown exponent $\alpha(>0)$ to represent its decay from the entrance $U(0)$ using

$$
U(x)=U(0)\left(1-\frac{x}{L_{s}}\right)^{\alpha} .
$$

Noting that $P\left(L_{S}\right)=0$ at the recirculation point and $a \approx L A I / h$ yields an expression for the onset of recirculation (normalized by canopy height $h$ ) as:

$$
\frac{L_{s}}{h}=(2 \alpha+1) \frac{P(0)}{U(0)^{2}} \frac{1}{C_{d} L A I} .
$$

While $\alpha$ remains unknown, it is possible to derive its lower bound by noting that just before the recirculation point, both $\partial U / \partial x \rightarrow 0$ and $\partial^{2} U / \partial x^{2} \rightarrow 0$ as $x \rightarrow L_{s}$. These velocity smoothness constraint arguments in the context of an integral momentum balance have a longstanding tradition in boundary-layer flows albeit for the vertical direction (e.g. the Karman-Pohlhausen method, see Panton 1984 p. 570).

We are primarily focusing on first-order and second-order smoothness because terms such as $\partial\left\langle u^{\prime} u^{\prime}\right\rangle_{y t} / \partial x$ in the mean momentum balance must remain small and bounded (relative to the drag force and pressure gradient) before $x \rightarrow L_{s}$. To illustrate why a small $\partial\left\langle u^{\prime} u^{\prime}\right\rangle_{y t} / \partial x$ can be used to derive a smoothness constraint on the mean velocity, consider the isotropic eddy-diffusivity $(K)$ first-order closure model for the Reynolds stresses given by

$$
\left\langle u_{i}^{\prime} u_{j}^{\prime}\right\rangle=-\frac{1}{2} K\left(\frac{\partial U_{i}}{\partial x_{j}}+\frac{\partial U_{j}}{\partial x_{i}}\right) .
$$

For such an elementary model, $\partial\left\langle u^{\prime} u^{\prime}\right\rangle_{y t} / \partial x$ necessitates smoothness in both $\partial U / \partial x$ and $\partial^{2} U / \partial x^{2}$. If such smoothness is not guaranteed, then modelled $\partial\left\langle u^{\prime} u^{\prime}\right\rangle_{y t} / \partial x$ from Eq. 12 may become a dominant term in Eq. 7 near $x \rightarrow L_{s}$ (inconsistent with LES). The smoothness requirements can be readily formulated by first computing $\partial U / \partial x$ and $\partial^{2} U / \partial x^{2}$,

$$
\begin{aligned}
& \frac{\partial U}{\partial x}=-\frac{\alpha U(0)\left(1-x / L_{s}\right)^{\alpha-1}}{L_{s}}, \\
& \frac{\partial^{2} U}{\partial x^{2}}=-\frac{\alpha(\alpha-1) U(0)\left(1-x / L_{s}\right)^{\alpha-2}}{L_{s}^{2}},
\end{aligned}
$$

and noting that as $x \rightarrow L_{s}, \alpha \geq 1$ and $\alpha \geq 2$, respectively, to maintain bounded first and second derivatives. Hence, this analysis guarantees that the lower value of $\alpha$ is 2 .

\subsection{Comparison between LES and Scaling Analysis: The Role of LAI}

The simplified model in Eq. 11 predicts the onset of recirculation inside the canopy given: (i) the boundary condition $P(0) / U(0)^{2}$, (ii) canopy drag attributes (i.e. $C_{d}$ ), and (iii) leaf area index. Noting that close to the ground $(z / h<0.1)$ for the LES runs reported here (see Figs. 7 and 10) $P(0) / U(0)^{2} \approx 2, L A I=8, C_{d}=0.2$, and by assuming $\alpha=2$ (its minimum value), Eq. 11 predicts that $L_{s} / h \geq 6$, in good agreement with LES results especially if to simplify the analysis we consider the constant density and sharp transition (see Fig. 7). 


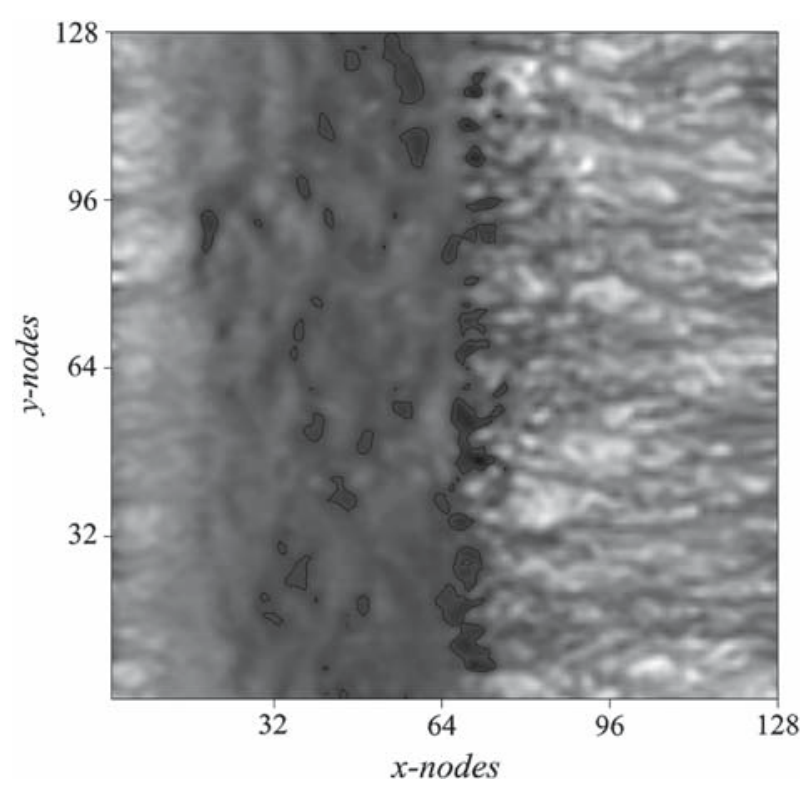

Fig. 13 Same as Fig. 4 a but for $\mathrm{LAI}=2$ and resolution $128 \times 128$

Furthermore, as LAI decreases from 8 to $6, L_{S} / h$ increases roughly by (8/6) or some $30 \%$, and this is consistent with the magnitude and directional shift in the LES runs as deduced from Fig. 6. We emphasize that the value of $P(0) / U(0)^{2}=2$ is not universal and is likely to depend on external factors such as the geostrophic wind, canopy height, and atmospheric stability.

\section{A Simplified Analysis for the Recirculation Outside the Canopy}

Thus far, it was demonstrated by the LES simulations that recirculation zones along the forest-to-clearing canopy edge are time intermittent consistent with earlier studies (e.g. Raupach et al. 1987; Flesch and Wilson 1999). Moreover, at the forest-to-clearing canopy edge, the recirculation and exit flow alternate in the spanwise direction (Fig. 4). Hence, a plausible hypothesise is that the volume fraction occupied by these two different flow structures must vary with the forest LAI. A qualitative confirmation comes from the comparison in Figs. 13 and $4 \mathrm{a}$. These figures represent horizontal sections of the instantaneous streamwise velocity close to the ground for $\mathrm{LAI}=2$ (Fig. 13) and $\mathrm{LAI}=8$ (Fig. 4a). For LAI $=2$, fewer (and smaller in size) negative velocity zones are present when compared to $\mathrm{LAI}=8$. It was earlier shown (see Fig. 6) that these zones do not generate any average recirculation in the mean velocity when $\mathrm{LAI}=2$. With the increase in $\mathrm{LAI}$, the negative velocity zones grow in number and size until they can generate mean negative velocities (consistent with an intermittent recirculation).

These spatial zones can also be interpreted as a relative time of existence of one state (i.e. recirculation or exit flow) at a single location. This implies that the BFS-like flow structure and the exit flow structure (see Fig. 14) occur with a relative frequency depending on the 

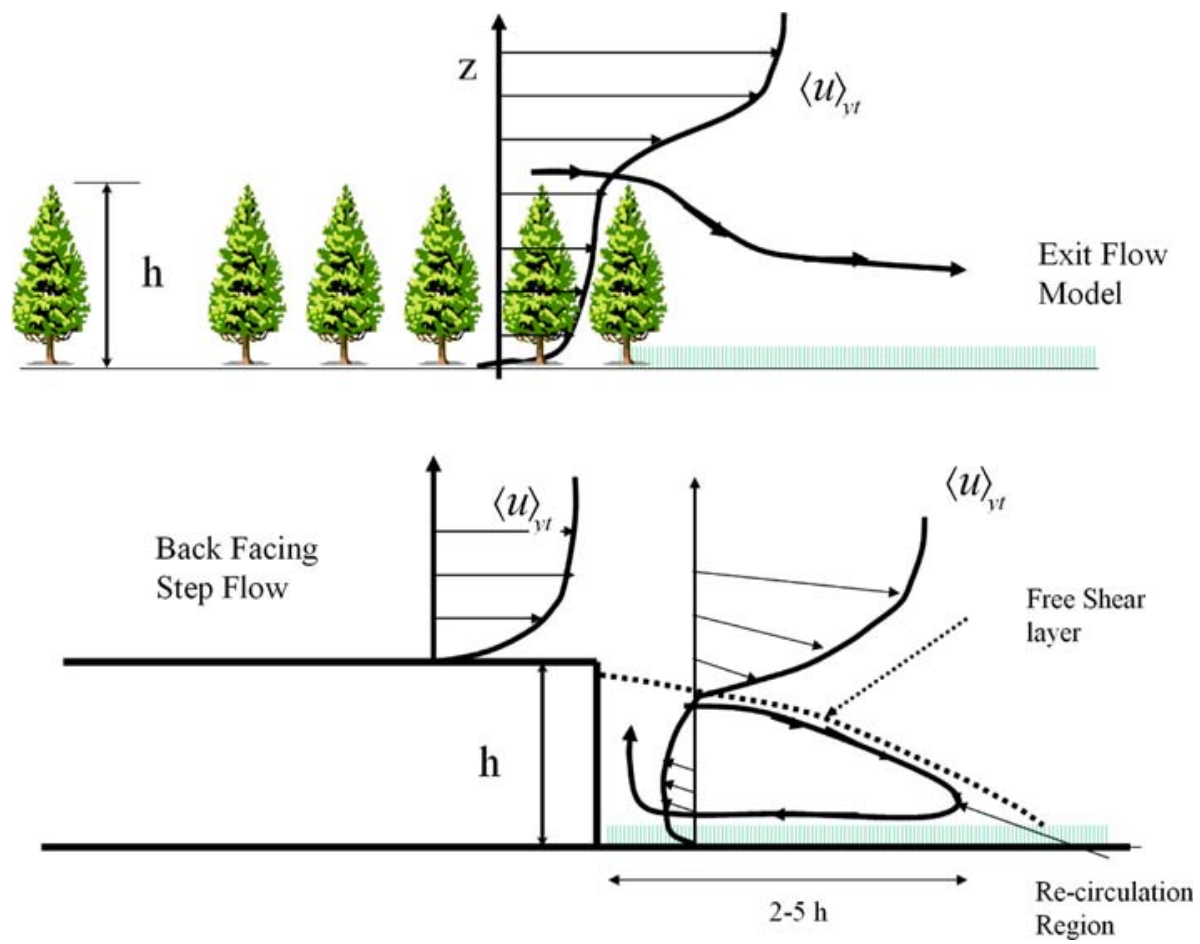

Fig. 14 Sketch of the two extreme flow cases considered in the superposition

forest LAI. To quantitatively analyze the relative contributions of these two flow structures, without the need of actually distinguishing between crosswind direction and time, we can define the sample fraction $\left(S f_{\bar{u}<0}(x, z)\right)$ as the number of measured samples with negative velocity divided by the total number of samples at a given $(x, z)$ location.

Figure 15 shows $S f_{\bar{u}<0}$ in the immediate vicinity of the forest-to-clearing transition for two different heights. From Fig. 15, $S f_{\bar{u}<0}$ increases with increasing LAI and, according to Fig. 4, $S f_{\bar{u}<0}$ decreases with increasing z. For reference, we included the estimates of time fraction of negative velocity obtained by Flesch and Wilson (1999) for their measurements in Manning (Alberta). As discussed in Sect. 3.2, Wilson and Flesch (1999) modelled this experiment with RANS using $C_{d} L A I \approx 3 / 4$, which approximately corresponds to our LAI $=4$ simulations. In fact, Fig. 15 (right) shows that measurements and LES results are similar, although the LES predicts a downwind decay of $S f_{\bar{u}<0}$ faster than the measurements. This, in turn, could be interpreted as a shorter recirculation. However, we did not attempt to simulate the exact experimental set-up and precise agreements should not be expected. An interesting point is that, according to our finding, $S f_{\bar{u}<0}$ of about 0.2 at $z / h=0.4$ corresponds to an inversion of the mean velocity component closer to the ground.

Next, we show how the mean flow can be expressed as a superposition of the two extremes: BFS-like flow and the exit flow, with the relative weights being some unknown function of LAI. The simulations available here were used to test this hypothesis for LAI $=4$ and 6 assuming the two 'end-members' are the mean flow with LAI $=2$ and 8 respectively. That is, for any position $(x, z)$ after the forest-to-clearing edge, the mean velocity can be decomposed into contributions originating from the BFS-like flow and exit flow using: 


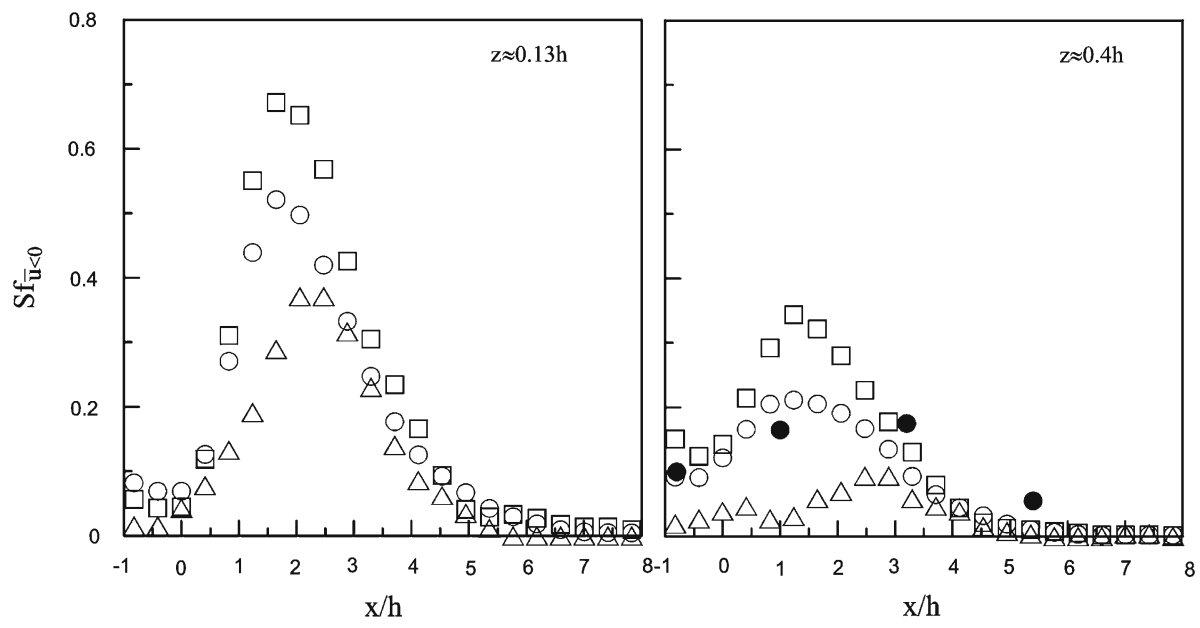

Fig. 15 Sample fraction with instantaneous resolved velocity lower than zero. Here $x / h$ is the normalized downwind distance relative to the forest-to-clearing transition. Solid symbols are for the Flesch and Wilson (1999) measurements. Open symbols are for simulations with LAI = 2 (open triangle), LAI = 4 (open circle), and $\mathrm{LAI}=8$ (open square)

$$
\langle\bar{u}(\mathrm{LAI})\rangle_{y t}=\left\langle\bar{u}\left(\mathrm{LAI}_{o}\right)\right\rangle_{y t}\left(\frac{\mathrm{LAI}_{b}-\mathrm{LAI}}{\Delta_{\mathrm{LAI}}}\right)^{\alpha_{1}}+\left\langle\bar{u}\left(\mathrm{LAI}_{b}\right)\right\rangle_{y t}\left(\frac{\mathrm{LAI}-\mathrm{LAI}_{o}}{\Delta_{\mathrm{LAI}}}\right)^{\alpha_{1}}
$$

Here $\mathrm{LAI}_{b}=8$ (i.e. large BFS-like flow contribution), $\mathrm{LAI}_{o}=2$ (large exit flow contribution), $\Delta_{\mathrm{LAI}}=\mathrm{LAI}_{b}-\mathrm{LAI}_{o}=6$, and $\alpha_{1}$ is an unknown exponent to be evaluated empirically from the LES runs here. An optimum $\alpha_{1}=0.38$ was determined based on the streamwise mean velocity component.

The scatter plot in Fig. 16a shows the comparison between the LES mean velocity profiles for the two extremes in the superposition model; $\mathrm{LAI}_{b}=8$ and $\mathrm{LAI}_{o}=2$. The scatter plot in Fig. 16b shows the predictions from the simplified model in Eq. 14 for the region $26 h<x<31 h$ and all $z<h$. The results support the scaling analysis reasonably well suggesting that such a linear superposition is reasonable. The scatter plot in Fig. 16c, d repeats the same comparison but for the mean vertical velocity using the same $\alpha_{1}=0.38$ derived from the longitudinal velocity component. Again, the good agreement independently confirms earlier arguments about the superposition given that the vertical velocity was not previously used in the estimation of $\alpha_{1}=0.38$.

\section{Summary and Conclusion}

How the forest canopy LAI controls the flow across a forest edge was investigated using one-way nested LES runs. This nesting eliminated the need for an unrealistic 'lid' near the canopy top. The structure of turbulence in the nested simulations was solved at two spatial resolutions: $8.2 \times 8.2 \times 1.7 \mathrm{~m}^{3}$ for the $128 \times 128$ simulations and $4.1 \times 4.1 \times 1.7 \mathrm{~m}^{3}$ for the $256 \times 256$ simulation. The results for the first-order and second-order statistics inside the forest canopy agree well with several field and laboratory experiments conducted for homogeneous dense canopies. 
(a)

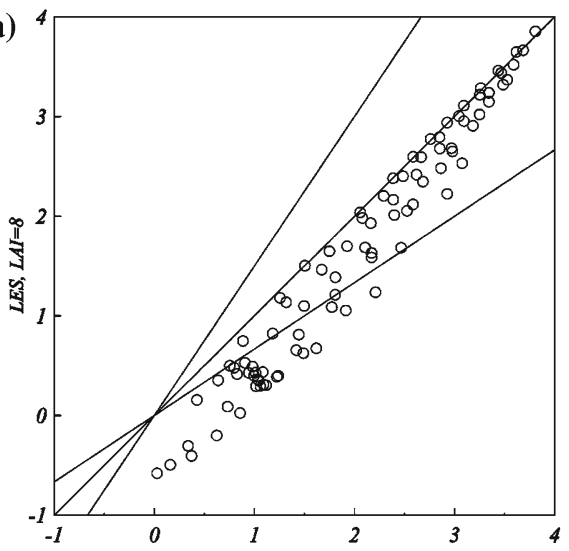

(c)

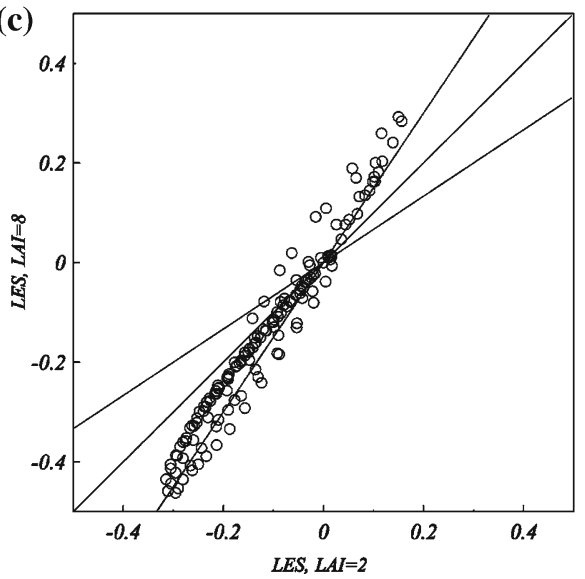

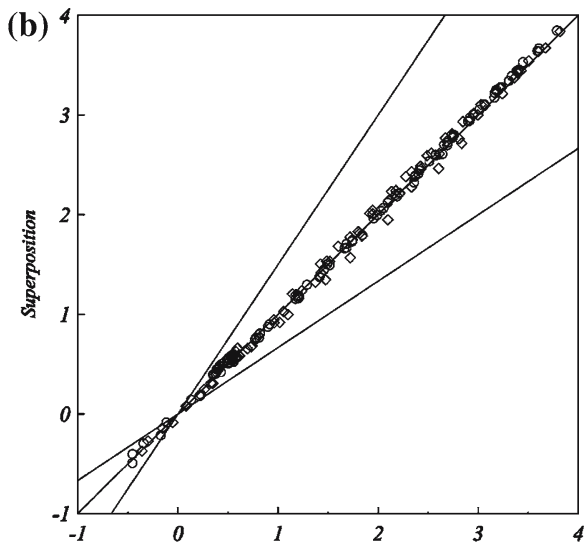

(d)

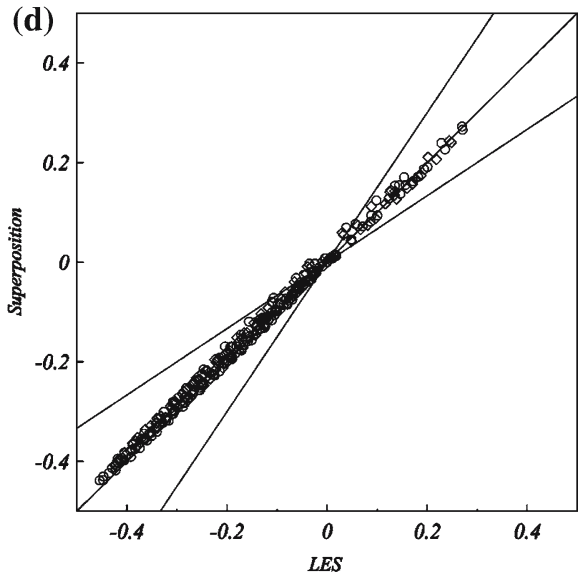

Fig. 16 Top panels: horizontal mean streamwise velocity $\langle u(x, z)\rangle_{y t}$ scaled with $u_{s 2}$ for $26 h<x<31 h$ and $z<h$. (a) Scatter plot of the two extreme of the superposition model; (b) Superposition model vs. LES simulations, $\mathrm{LAI}=4$ (open diamonds) and 6 (open circle). Bottom panels: vertical mean velocity $\langle w(x, z)\rangle_{y t}$ scaled with $u_{s 2}$ for $26 h<x<31 h$ and $z<h$; (c) Scatter plot of the two extreme of the superposition model. (b) Superposition model vs. LES simulations, LAI $=4$ (open diamonds) and 6 (open circle). The off diagonal lines bound the $50 \%$ errors

Using LES runs, it was shown that by increasing the forest LAI, the mean flow properties both within the forest and in the clearing near the forest edge are altered in two fundamental ways. Near the forest edge and into the clearing, a recirculation zone sets up. Deep inside the forest, another recirculation zone sets up as the flow exits the clearing and enters the tall forest canopy.

At the forest-to-clearing edge, the BFS-like effects do not lead to a continuous rotor-like circulation but to intermittent motion whose frequency and size increase with increasing forest LAI. However, beyond an LAI $=6$, increasing LAI has minor impacts on this intermittent motion. The fraction of time with velocity reversal (due to the intermittent negative velocity) at the forest-to-clearing transition approximately agrees with the measurements of Flesch and Wilson (1999). Also, a mean recirculation is predicted accordingly to the RANS model calculations reported in Flesch and Wilson (1999). These comparisons lend confidence to the LES results. 
Inside the forest canopy, the horizontal distance at which a recirculation zone begins was well reproduced using a longitudinal integral momentum balance between the mean pressure gradient and the drag force. Both the analytical model and the LES runs showed that increasing LAI shifted the onset of the recirculation closer to the inflow forest edge. Furthermore, this recirculating motion cannot be attributed to a turbulent effect because its longitudinal size exceeds typical canopy turbulence length scales (e.g. Poggi et al. 2004).

The ecological significance of these recirculation zones is now the subject of an on-going investigation and will require the addition of dispersion of scalars (e.g. Cassiani et al. 2005, 2007a, b), and solid particles via Lagrangian trajectories driven by the LES motions. A recirculation zone near the forest edge can enhance seed and pollen deposition in gaps, and hence, disproportionately affect gap colonization. Furthermore, a recirculation zone inside the canopy may enhance re-suspension of seeds and pollen from the forest floor, and hence, increase their probability of uplifting outside the canopy volume. This uplifting was shown to be a necessary condition for long-distance dispersal (e.g. Nathan et al. 2002; Katul et al. 2005).

Acknowledgements We acknowledge the numerous comments by an anonymous reviewer which greatly improved the LES evaluation here. Cassiani and Albertson acknowledge the support by the U.S. Department of Energy's Office of Science (BER) through the Southeastern Regional Center of the National Institute for Climatic Change Research at Duke University. Katul acknowledges partial support from the National Science Foundation (NSF-EAR-0635787 and NSF-EAR-06028432), from the United States Department of Agriculture (USDA) Biotechnology Risk Assessment Program (BRAP-2005-39454-16457), and from the Binational Agricultural Research and Development Fund (BARD, Grant \# IS-3861-06).

\section{References}

Albertson JD (1996) Large-eddy simulation of Land-Atmosphere Interaction. Ph.D. thesis, university of California Davis, $185 \mathrm{pp}$

Albertson JD, Parlange MB (1999a) Surface length scales and shear stress: implications for land-atmosphere interaction over complex terrain. Water Resour Res 35:2121-2132

Albertson JD, Parlange MB (1999b) Natural integration of scalar fluxes from complex terrain. Adv Water Resour 23:239-252

Albertson JD, Katul, Wiberg P (2001) Relative importance of local and regional controls on coupled water, carbon, and energy fluxes. Adv Water Resour 24(9-10):1103-1118

Amiro BD (1990) Comparison of turbulence statistics within 3 Boreal forest canopies. Boundary-Layer Meteorol 51:99-121

Antonia RA, Luxton RE (1974) Characteristics of turbulence within an internal boundary layer. In: Frenkiel FN, Munn RE (eds) Turbulent diffusion in environmental pollution (Adv. Geophys. 18A). Academic Press, pp 263-285

Armali F, Durst F, Pereira JCF, Schonung B (1983) Experimental and theoretical investigation of backward facing step flow. J Fluid Mech 127:473-496

Asner GP, Jonathan MO, Hicke JA (2003) Global synthesis of leaf area index observations: implications for ecological and remote sensing studies. Global Ecol Biogeogr 12(3):191-205

Bergen JD (1975) Air movement in a forest clearing as indicated by smoke drift. Agric Meteorol 15:165-179

Canuto C, Hussaini MY, Quarteroni A, Zang TA (1988) Spectral methods in fluid dynamics. Springer Verlag, Berlin, $557 \mathrm{pp}$

Cassiani M, Radicchi A, Giostra U (2005) Probability density function modelling of concentration in and above a canopy layer. Agric For Meteorol 133:153-165

Cassiani M, Radicchi A, Albertson JD (2007a) Modelling of concentration fluctuations in canopy turbulence. Boundary-Layer Meteorol 122(3):655-681

Cassiani M, Radicchi A, Albertson JD, Giostra U (2007b) An efficient algorithm for scalar PDF modelling in incompressible turbulent flow; numerical analysis with evaluation of IEM and IECM micro-mixing models. J Comp Phys 223(2):519-550 
Chen JM, Clack TA, Novak MD, Adams RS (1995) A wind tunnel study of turbulent airflow in forest clear cuts. In: Coutts MP, Grace J (eds) Wind and Trees, Chap. 4. Cambridge University Press, London

Chun KB, Sung HJ (1998) Visualization of a locally-forced separated flow over a backward-facing step. Exp Fluids 25:133-142

Dejoan A, Leschziner MA (2004) Large-eddy simulation of periodically perturbed separated flow over a backward-facing step. Int J Heat Fluid Fl 25:581-559

Deardorff JW (1970) A numerical study of three-dimensional turbulent channel flow at large Reynolds numbers. Investigations of neutral and unstable planetary boundary layers. J Fluid Mech 41:453-465

Deardorff JW (1972) Numerical investigations of neutral and unstable planetary boundary layers. J Atmos Sci 29:91-115

Detto M, Katul GG, Siqueira MB, Juang J-Y, Stoy P (2007) The structure of turbulence near a tall forest edge: the backward facing step flow analogy revisited. Ecol Appl (in press)

Dwyer MJ, Patton EG, Shaw RH (1997) Turbulent kinetic energy budgets from a large-eddy simulation of airflow above and within a forest canopy. Boundary-Layer Meteorol 84:23-43

Ellsworth DS, Oren R, Huang C, Phillips N, Hendrey RH (1995) Leaf and canopy responses to elevated CO2 in a pine forest under free-air CO2 enrichment. Oecologia 104:139-146

Flesch TK, Wilson JD (1999) Wind and remnant tree sway in forest cutblocks. I. Measured winds in experimental cutblocks. Agric For Meteorol 93:229-242

Furuichi N, Hachiga T, Kumada M (2004) An experimental investigation of a large-scale structure of a two-dimensional backward-facing step by using advanced multi-point LDV. Exp Fluids 36:274-281

Garratt JR (1990) The internal boundary layer: a review. Boundary-Layer Meteorol 50:171-203

Germano M (1992) Turbulence: the filtering approach. J Fluid Mech 238:325-336

Germano M, Piomelli U, Moin P, Cabot WH (1991) A dynamical subgrid-scale eddy viscosity model. Phys Fluids A 3(7):1760-1765

Gullbrand J, Chow FK (2003) The effect of numerical errors and turbulence models in large-eddy simulations of channel flow, with and without explicit filtering. J Fluid Mech 495:323-341

Holland JZ (1989) On pressure-driven wind in deep forests. J Appl Meteorol 28:1349-1355

Irvine MR, Gardiner BA, Hill MK (1997) The evolution of turbulence across a forest edge. Boundary-Layer Meteorol 84:467-496

Judd MJ, Raupach MR, Finnigan JJ (1996) A wind tunnel study of turbulent flow around single and multiple windbreaks. 1. Velocity fields. Boundary-Layer Meteorol 80(1-2):127-165

Kaltenbach HJ (1997) Cell aspect ratio dependence of anisotropy measures for resolved and subgrid scale stresses. J Comput Phys 136(2):399-410

Katul GG, Albertson JD (1998) An investigation of higher-order closure models for a forested canopy. Boundary-Layer Meteorol 89(1):47-74

Katul GG, Chang WH (1999) Principal length scales in second-order closure models for canopy turbulence. J Appl Meteorol 38:1631-1643

Katul GG, Leuning R, Kim J, Denmead OT, Miyata A, Harazono Y (2001) Estimating CO2 Source/Sink distributions within a rice canopy using higher-order closure models. Boundary-Layer Meteorol 98:103125

Katul GG, Mahrt L, Poggi, Sanz C (2004) One and two equation models for canopy turbulence. BoundaryLayer Meteorol 113:81-109

Katul GG, Porporato A, Nathan R, Siqueira M, Soons MB, Poggi D, Horn HS, Levin SA (2005) Mechanistic analytical models for long-distance seed dispersal by wind. Amer Nat 166:369-376

Khanna S, Brasseur JG (1997) Analysis of Monin-Obukhov similarity from large-eddy simulation. J Fluid Mech 345:251-286

Klaassen W (1992) Average fluxes from heterogeneous vegetated regions. Boundary-layer Meteorol 58:329_ 354

Kostas J, Soria J, Chong MS (2002) Particle image velocimetry measurements of a backward-facing step flow. Exp Fluids 33:838-853

Kosovic B (1997) Subgrid-scale modelling for the large-eddy simulation of high-Reynolds-number boundary layers. J Fluid Mech 336:151-182

Laurence W (2004) Forest-Climate interactions in fragmented tropical landscapes. Phil Trans Roy Soc London 359:345-352

Lesieur M, Metais O, Comte P (2005) Large-eddy simulation of turbulence. Cambridge University Press, Cambridge, 219 pp

Li Z, Lin JD, Miller DR (1990) Air flow over and trough a forest edge: steady state numerical simulation. Boundary-Layer Meteorol 51:179-197

Liu J, Chen JM, Black TA, Novak MD (1996) E- $\varepsilon$ modelling of turbulent air flow downwind of a model forest edge. Boundary-Layer Meteorol 77:21-44 
Mason PJ, Thomson DJ (1987) Large-eddy simulations of the neutral-static-stability planetary boundary layer. Quart J Roy Meteorol Soc 113(476):413-443

Mason PJ, Thomson DJ (1992) Stochastic backscatter in large-eddy simulation of boundary layer. J Fluid Mech 242:51-78

Meyers TP, Baldocchi DD (1991) The budgets of turbulent kinetic-energy and Reynolds stress within and above a deciduous forest. Agric For Meteorol 53:207-222

Moeng CH (1984) A large-eddy simulation model for the study of planetary boundary-layer turbulence. J Atmos Sci 41:2052-2062

Moin P, Reynolds WC, Ferziger JH (1978) Large-eddy simulation of incompressible turbulent channel flow. Dept. Mech. Eng. Stanford Universiy, Rep. TF-12

Morse AP, Gardiner BA, Marshall BJ (2002) Mechanisms controlling turbulence development across a forest edge. Boundary-Layer Meteorol 103:227-251

Nakagawa H, Nezu I (1987) Experimental investigation on turbulent structure of a backward facing step flow in an open channel. J Hydraul Res 25:67-88

Nathan R, Katul GG, Horn HS, Thomas SM, Oren R, Avissar R, Pacala SW, Levin SA (2002) Mechanisms of long-distance dispersal of seeds by wind. Nature 418:409-413

Nezu I (2005) Open-channel flow turbulence and its research prospect in the 21st century. J Hydraul Eng 131:229-246

Orlandi P (2000) Fluid flow phenomena. Kluwer Academic Publisher, Dordrecht, 356 pp

Orszag SA, Pao YH (1974) Numerical computation of turbulent shear flows. Adv Geophys 18A:224-236

Panton R (1984) Incompressible flows. John Wiley and Sons, New York, 779 pp

Patton EG, Shaw RH, Judd MJ, Raupach MR (1998) Large-eddy simulation of windbreak flow. BoundaryLayer Meteorol 87:275-306

Patton EG, Davis KJ, Barth MC, Sullivan PP (2001) Decaying scalars emitted by a forest canopy: a numerical study. Boundary-Layer Meteorol 100:91-129

Poggi D, Porporato A, Ridolfi L, Albertson JD, Katul GG (2004) The effect of vegetation density on canopy sublayer turbulence. Boundary-Layer Meteorol 111:565-587

Porte-Agel F, Meneveau C, Parlange MB (2000) A scale dependent dynamic model for large-eddy simulation: application to a neutral atmospheric boundary layer. J Fluid Mech 415:261-284

Piirto M, Saarenrinne P, Eloranta H, Karvinen R (2003) Measuring turbulence energy with PIV in a backward-facing step flow. Exp Fluids 35:219-236

Rao KS, Wyngaard JC, Cote OR (1974) The structure of the two- dimensional internal boundary layer over sudden change of surface roughness. J Atmos Sci 31:738-746

Raupach MR, Bradley EF, Ghadiri H (1987) Wind tunnel investigation into the aerodynamic effect of forest clearing of the nesting of Abbott's Booby on Christmas Island. Progress Report, CSIRO Division of Environmental Mechanics, GPO Box 821, Canberra, ACT 2601, Australia, Canberra

Raynor GS (1971) Wind and temperature structure in a coniferous forest and a contiguous field. Forest Sci $17: 351-363$

Sagaut P (2002) Large-eddy simulation for incompressible flows. Springer Verlag, Berlin, 426 pp

Scarano F, Benocci C, Riethmuller ML (1999) Pattern recognition analysis of the turbulent flow past a backward facing step. Phys Fluids 11:3808-3818

Scotti A, Meneveau C, Lilly DK (1993) Generalized smagorinsky model for anisotropic grids. Phys Fluids 5(7):2306-2308

Schram C, Rambaud P, Riethmuller ML (2004) Wavelet based eddy structure deduction from a backward facing step flow investigated using particle image velocimetry. Exp Fluids 36:233-245

Schmidt RH, Schumann U (1989) Coherent structure of the convective boundary-layer derived from largeeddy simulations. J Fluid Mech 200:511-562

Shaw RH, Patton EG (2003) Canopy element influences on resolved- and subgrid scale energy within a largeeddy simulation. Agric For Meteorol 115:5-17

Shaw RH, Schumann U (1992) Large-eddy simulation of turbulent flow above and within a forest. BoundaryLayer Meteorol 61:47-64

Shen SH, Leclerc MY (1997) Modelling the turbulence structure in the canopy layer. Agric For Meteorol 87:3-25

Sheu TWH, Rani HP (2006) Exploration of vortex dynamics for transitional flows in a three-dimensional backward-facing step channel. J Fluid Mech 550:61-83

Simpson R (1989) Turbulent boundary layer separation. Ann Rev Fluid Mech 21:205-234

Spazzini PG, Iuso G, Onorato M, Zurlo N, Cicca GMD (2001) Unsteady behaviour of backfacing step flow. Exp Fluids 30:551-561 
Su HB, Shaw RH, Paw U KT, Moeng CH, Sullivan PP (1998) Turbulent statistics of neutrally stratified flow within and above a sparse forest from large-eddy simulation and field observations. Boundary-Layer Meteorol 88:363-397

Su HB, Shaw RH, Paw U KT (2000) Two-point correlation analysis of neutrally stratified flow within and above a forest from large-eddy simulation. Boundary-Layer Meteorol 94:423-460

Smagorinsky J (1963) General circulation experiments with the primitive equations. I: the basic experiment. Mon Wea Rev 91(3):99-165

Sullivan PP, McWilliams JC, Moeng CH (1996) A grid nesting algorithm for large-eddy simulation of planetary boundary-layer flows. Boundary-Layer Meteorol 80:167-202

Watanabe T (2004) Large-eddy simulation of coherent turbulence structures associated with scalar ramps over plant canopies. Boundary-Layer Meteorol 112:307-341

Wilson JD, Ward DP, Thurtell DW, Kidd GE (1982) Statistics of atmospheric turbulence within and above a corn canopy. Boundary-Layer Meteorol 24:495-519

Wilson JD, Flesch TK (1999) Wind and remnant tree sway in forest cutblocks. III. A windflow model to diagnose spatial variation. Agric For Meteorol 93:259-282

Xie Z, Voke PR, Hayden P, Robins GA (2004) Large-eddy simulations of turbulent flow over rough surface. Boundary-Layer Meteorol 111:417-440

Yang B, Raupach MR, Shaw RH, Paw KT, Morse A (2006a) Large-eddy simulation of turbulent flow across a forest edge. Part I: flow statistics. Boundary-Layer Meteorol 120:377-412

Yang B, Morse A, Shaw RH, Paw U KT (2006b) Large-eddy simulation of turbulent flow across a forest edge. Part II: momentum and turbulent kinetic energy budget. Boundary-Layer Meteorol 121:433-457 